\title{
PAPER \\ Multi-View 3D CG Image Quality Assessment for Contrast Enhancement Based on S-CIELAB Color Space
}

\author{
Norifumi KAWABATA ${ }^{\dagger a)}$, Student Member and Masaru MIYAO ${ }^{\dagger b)}$, Member $^{\text {(bem }}$
}

\begin{abstract}
SUMMARY Previously, it is not obvious to what extent was accepted for the assessors when we see the 3D image (including multi-view 3D) which the luminance change may affect the stereoscopic effect and assessment generally. We think that we can conduct a general evaluation, along with a subjective evaluation, of the luminance component using both the S-CIELAB color space and CIEDE2000. In this study, first, we performed three types of subjective evaluation experiments for contrast enhancement in an image by using the eight viewpoints parallax barrier method. Next, we analyzed the results statistically by using a support vector machine (SVM). Further, we objectively evaluated the luminance value measurement by using CIEDE2000 in the S-CIELAB color space. Then, we checked whether the objective evaluation value was related to the subjective evaluation value. From results, we were able to see the characteristic relationship between subjective assessment and objective assessment.

key words: multi-view $3 D$ image, Double Stimulus Impairment Scale (DSIS), Luminance, S-CIELAB color space, CIEDE2000, Support Vector Machine (SVM)
\end{abstract}

\section{Introduction}

With the increasing popularity of Quad Full HDTV (QFHDTV), the 3D still image or video quality has become better than that of Full HDTV (FHDTV). Researchers have focused on 3D videos as an added value. Further, advancements in the research and development of multi-view 3D image quality assessments are expected in the near future.

In the past, many studies were conducted on still image [1]-[5] or video [6]-[8] quality assessment using the SCIELAB color space, CIEDE2000, considering the spatial frequency characteristics of the human visual system. On the other hand, there was study for measurement of the gazing information by using S-CIELAB color space [9]. However, in a 3D still image, luminance may affect the stereoscopic effect or the quality assessment and we have not yet determined the amount of luminance required to see a binocular or a multi-view 3D image. In this study, we focused not on the 2D still images but on the multi-view 3D images including the contrast enhancement in order to clarify the relation among multi-view stereoscopic effect, luminance, and color difference as one of novelty. We consider that we need to clear the relation between the coded image quality and the contrast enhancement in order to construct better view-

Manuscript received September 2, 2016.

Manuscript revised February 5, 2017.

Manuscript publicized March 28, 2017.

${ }^{\dagger}$ The authors are with the Graduate School of Information Science, Nagoya University, Nagoya-shi, 464-8603 Japan.

a)E-mail: norifumi@nagoya-u.jp

b)E-mail:miyao@nagoya-u.jp

DOI: $10.1587 /$ transinf.2016EDP7367 ing environment. In order to see a multi-view 3D video anytime and at any place, first, we need to experimentally verify both subjective and objective assessments. With respect to the subjective assessment, we need to verify whether the assessors can perceive the amount of luminance requires in a case when the luminance is changed. With respect to the objective assessment, we need to verify the adaptive contrast or luminance of a multi-view 3D image by using the S-CIELAB color space. By quantifying these assessments, we believe that a general assessment is possible.

On the other hand, as represented by the region of interest (ROI), we need to know the 3D image region that the assessors are interested in when they see the 3D image, and thus far, there have been many studies on an image quality evaluation for the look-at point. In the past, we verified through a subjective quality evaluation experiment of 3D CG images in the case of coded degradation by regions with the eight viewpoints parallax barrier method [10] and found the evaluation value for the object region of an image, which is affected by the type of contents. However, for the color information, we mainly judged by eyesight. Thus, our results may not sufficient for evaluating a multi-view 3D CG color image. Therefore, we enable to evaluate exactly more and more, considering both color difference and luminance difference. We considered that we need to verify carrying out the evaluation experiment to what extent was not interfered with regard to the $3 \mathrm{D} C \mathrm{CG}$ images including the contrast enhancement and the coded degradation of the object region (We consider that the coded degradation of the object region easily affects the evaluation).

There have been many studies on the subjective evaluation of the 3D image or video quality [7]-[12]. However, to the best of our knowledge, there have been no reports on the relationship between luminance and coded degradation. In ITU-R, they report the standardization of binocular 3D video quality evaluations [15]. However, a common assessment standard necessary for viewing $3 \mathrm{D}$ videos, which has not defined internationally irrespective of whether the viewing is glassless or with glasses.

In this study, we investigated the following:

1) Not considering the contrast enhancement (Exp. 1): First, we encoded and decoded the original image by H.265/HEVC. Then, we carried out a subjective quality evaluation of the coded 3D CG image with the eight viewpoints parallax barrier method (Exp. 1-1). Next, we analyzed the results statistically and classified the 
subjective evaluation value by using SVM (Exp. 1-1). We then objectively analyzed the luminance component $L^{*}$ of the generated 3D CG image by transforming the S-CIELAB color space (Exp. 1-2). Next, we compared the original image to the coded image by using CIEDE2000 and analyzed this result objectively (Exp. 1-3) [4].

2) For the contrast enhancement of all regions (Exp. 2): First, we processed the contrast enhancement for all regions of a 3D CG image by using adaptive histogram equalization (AHE). Then, we encoded and decoded the processing image by H.265/HEVC. Next, we conducted the same subjective quality evaluation as that in Exp. 1-1 (Exp. 2-1). Thereafter, we analyzed and classified the results statistically as in Exp. 1-1 (Exp. 2-1). We then objectively analyzed the luminance component $L^{*}$ of the generated 3D CG image as in Exp. 12 (Exp. 2-2) and compared the original image to the coded image as in Exp. 1-3 (Exp. 2-3) [13].

3) For the contrast enhancement of the object region (Exp. 3): First, we processed the contrast enhancement for the object region of a 3D CG image by using AHE. Then, we carried out the same process as in Exps. 1-1 and 2-1 (Exp. 3-1). Next, we objectively analyzed the luminance component $L^{*}$ of the generated 3D CG image as in Exps. 1-2 and 2-2 (Exp. 3-2). We compared the original image to the coded image as in Exps. 1-3 and 2-3 (Exp. 3-3) [14].

On the basis of the experimental results, we analyzed and considered the relationship among the 3 experiments.

As composition in this study, first, in Sec. 1, we described the introduction for the background and purpose of research. Next, in Sec. 2, we will describe about methods for the image quality evaluation in this study. In Sec. 3, we will describe about the result of Exp. 1 and discussion. In Sec. 4, we will describe about the result of Exp. 2 and discussion. In Sec. 5, we will describe about the result of Exp. 3 and discussion. Finally, in Sec. 6, we will describe about the conclusion and the future work.

\section{Image Quality Evaluation}

\subsection{D CG Image Contents in this Study}

In this study, we prepared the 3D CG image contents for the three experiments. In Exp. 1, we used the 3D CG images shown in Fig. 1, called "Museum Figs. 1 (1a) and (1b)" and "Wonder World Figs. 1 (1c) and (1d)," provided by NICT free of charge [16]. Figures 1 (1a) and (1b) show the reference images. Figures 1 (1c) and (1d) show the coded images. In Exp. 2, we used the 3D CG images shown in Fig. 2, called "Museum Figs. 2 (2a), (2c), and (2e)" and "Wonder World Figs. 2 (2b), (2d), and (2f)" [16]. Figures 2 (2a) and (2b) show the reference images. Figures 2 (2c) and (2d) show the reference images after the AHE processing of all regions. Figures $2(2 \mathrm{e})$ and $(2 \mathrm{f})$ show the coded images after the AHE processing of all regions. In Exp. 3, we used

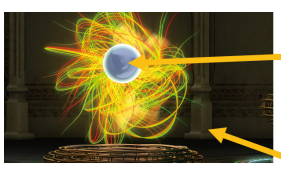

(1a) Museum $(Q P=$ ref $)$

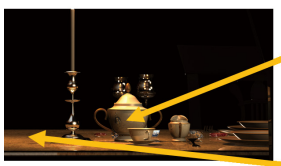

(1c) Wonder World $(Q P=r e f)$
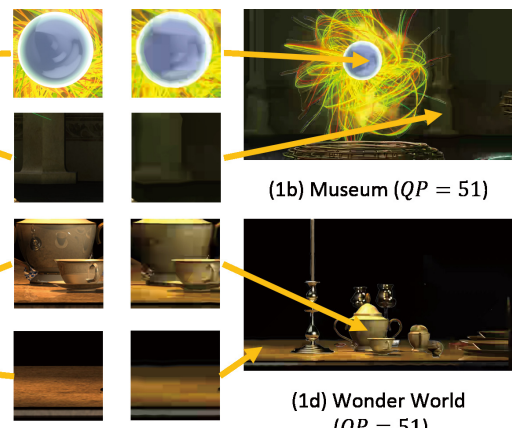

(1b) Museum ( $Q P=51)$

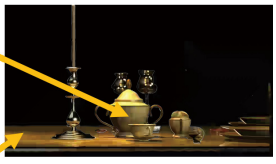

(1d) Wonder World $(Q P=51)$
Fig. 1 3D CG image contents used in the Exp. 1

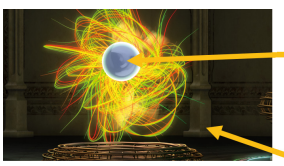

(2a) Museum $(Q P=$ ref $)$

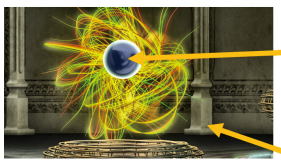

(2c) Museum_ah $(Q P=r e f)$

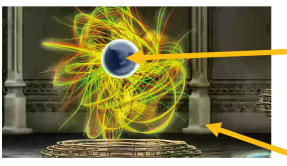

(2e) Museum_ah $(Q P=51)$
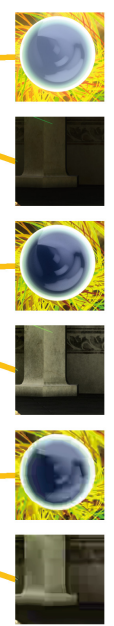

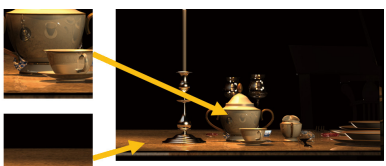

(2b) Wonder World $(Q P=r e f)$
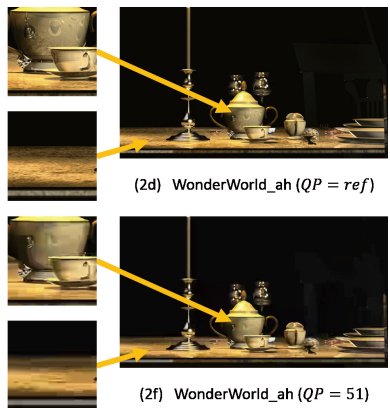

Fig. 2 3D CG image contents used in the Exp. 2

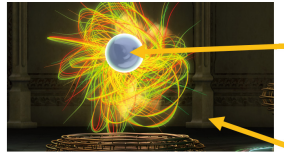

(3a) Museum $\left(Q P_{o b j}=r e f\right)$

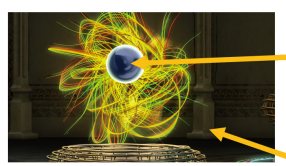

(3c) Museum_ah $\left(Q P_{o b j}=r e f\right)$

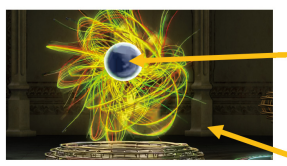

(3e) Museum_ah $\left(Q P_{o b j}=51\right)$
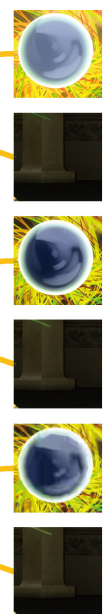
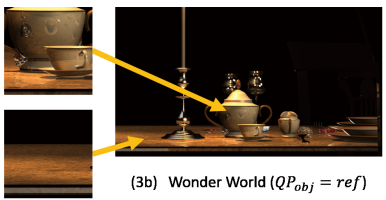

(3b) Wonder World $\left(Q P_{o b j}=r e f\right)$
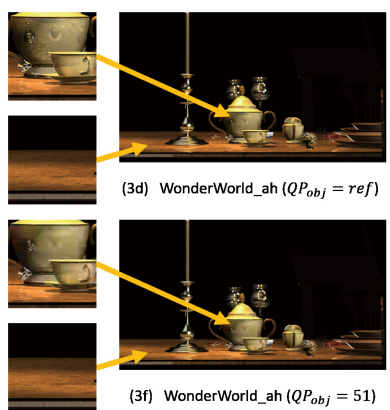

Fig. 3 3D CG image contents used in the Exp. 3

the 3D CG images shown in Fig. 3, called "Museum Figs. 3 (3a), (3c), and (3e)" and "Wonder World Figs. 3 (3b), (3d), and (3f)" [16]. Figures 3 (3a) and (3b) show the reference images. Figures $3(3 \mathrm{c})$ and (3d) show the reference images after the AHE processing of the object region. Figures 3 (3e) and (3f) show the coded images after the AHE processing of the object region. 


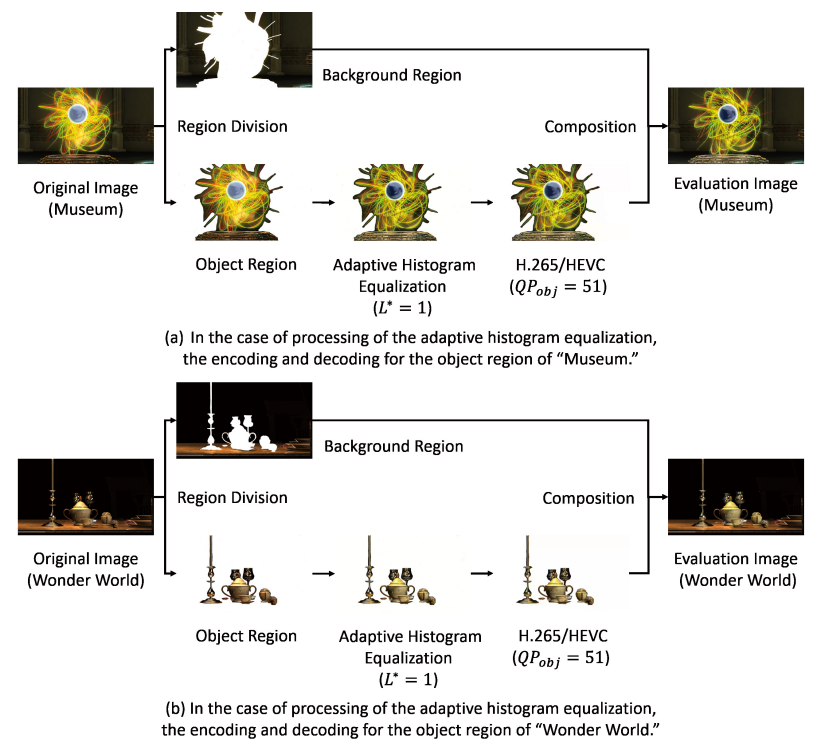

Fig. 4 Example of creating the evaluation image (Exp. 3)

Next, the process to generate $3 \mathrm{D}$ images can be described as follows:

1) First, we constructed eight viewpoints $C G$ cameras in a 3D CG space. Then, we processed the camera work and rendering. As a result, we could generate eight viewpoints CG cameras.

2) Then, in Exp. 2, we conducted the processing for all regions to the contrast enhancement by AHE on definition of maximum luminance value commonly by each viewpoint. In Exp. 3, we processed for the object region to the contrast enhancement by AHE on definition of maximum luminance value commonly by each viewpoint (as shown in Fig. 4). In Exp. 1, we did not process the contrast enhancement by using AHE.

3) Then, we encoded and decoded the generated $3 \mathrm{D}$ image by using H.265/HEVC. Further, in Exp. 3, we encoded and decoded only the object region of the generated 3D image by using H.265/HEVC.

4) After repeating these processes for eight viewpoints, we composed images for each viewpoint. Consequently, the 3D images were generated.

With respect to the types of image content, in Exp. 1, we prepared 14 sequences of 3D CG images, including the quantization parameter (QP) of H.265/HEVC and the contents ("Museum" and "Wonder World"). In Exp. 2, we prepared 52 sequences of 3D CG images, including the QP of H.265/HEVC, the maximum luminance parameter $(L)$ of AHE, and the contents ("Museum" and "Wonder World"). In Exp. 3, we prepared 52 sequences of 3D CG images, including the QP of H.265/HEVC, the maximum luminance parameter $(L)$ of AHE, and the contents ("Museum" and "Wonder World"). However, in Exp. 3, we focused on the object region of the 3D CG images. Therefore, we did not process the background region of the images.

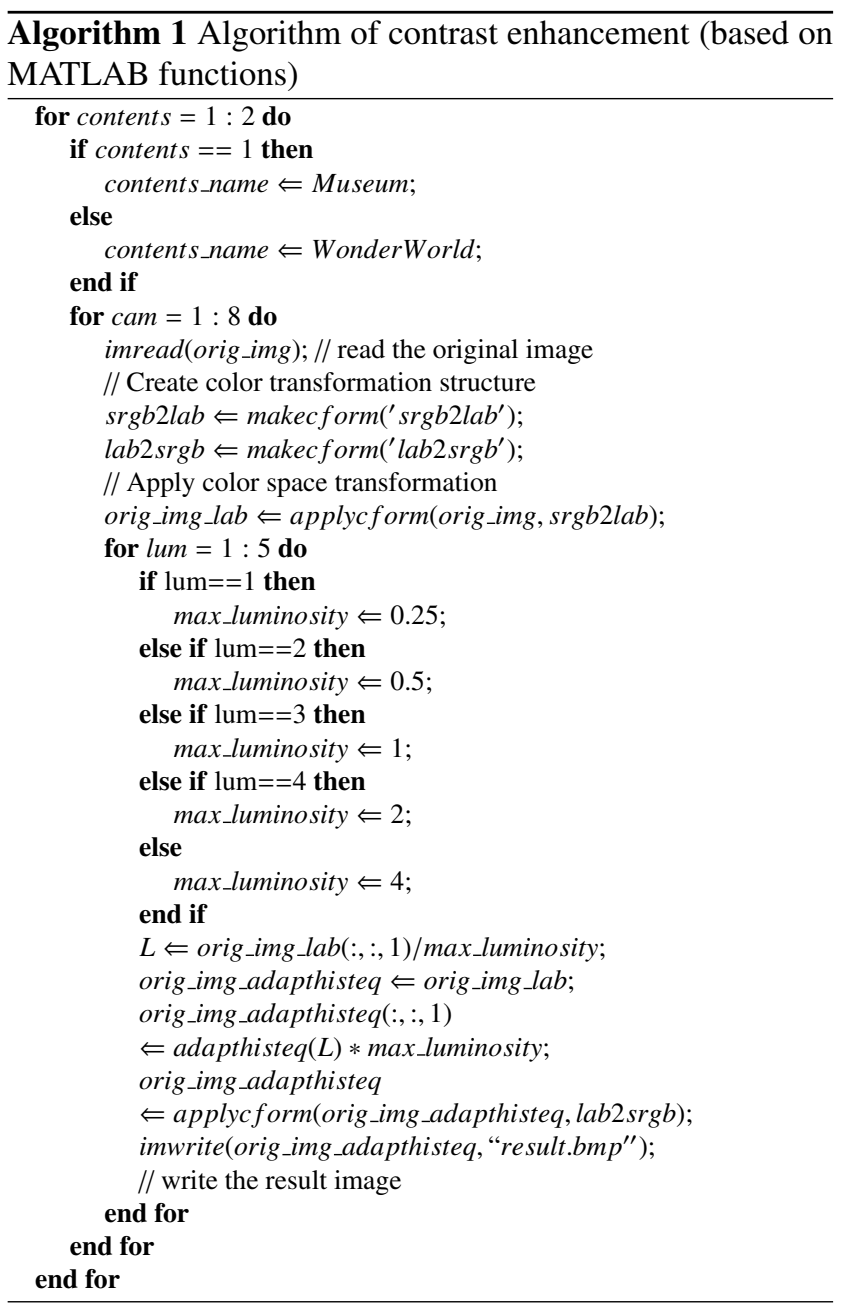

\subsection{Processing of the Image Contrast Enhancement}

In this study, we processed the image contrast enhancement by using the algorithm shown in Algorithm 1. Algorithm 1 shows the generation of multi-view images from the reading and processing of images to the saving of images.

\subsection{S-CIELAB Color Space and CIEDE2000}

Figure 5 shows the flowchart for the process from loading an image to transforming the S-CIELAB color space and CIEDE2000 [2]. We have explained the S-CIELAB color space and CIEDE2000 in Appendices A and B, therefore, please see the explanation of Appendices A and B in detail [2]. We show briefly from the following. Finally, $L^{*}, a^{*}$, and $b^{*}$ are shown in Eq. (1)-(3).

$$
L^{*}= \begin{cases}116\left(\frac{Y}{Y_{n}}\right)^{\frac{1}{3}}-16 & \left(\frac{Y}{Y_{n}}>0.008856\right) \\ 903.3\left(\frac{Y}{Y_{n}}\right) & \text { (otherwise) }\end{cases}
$$




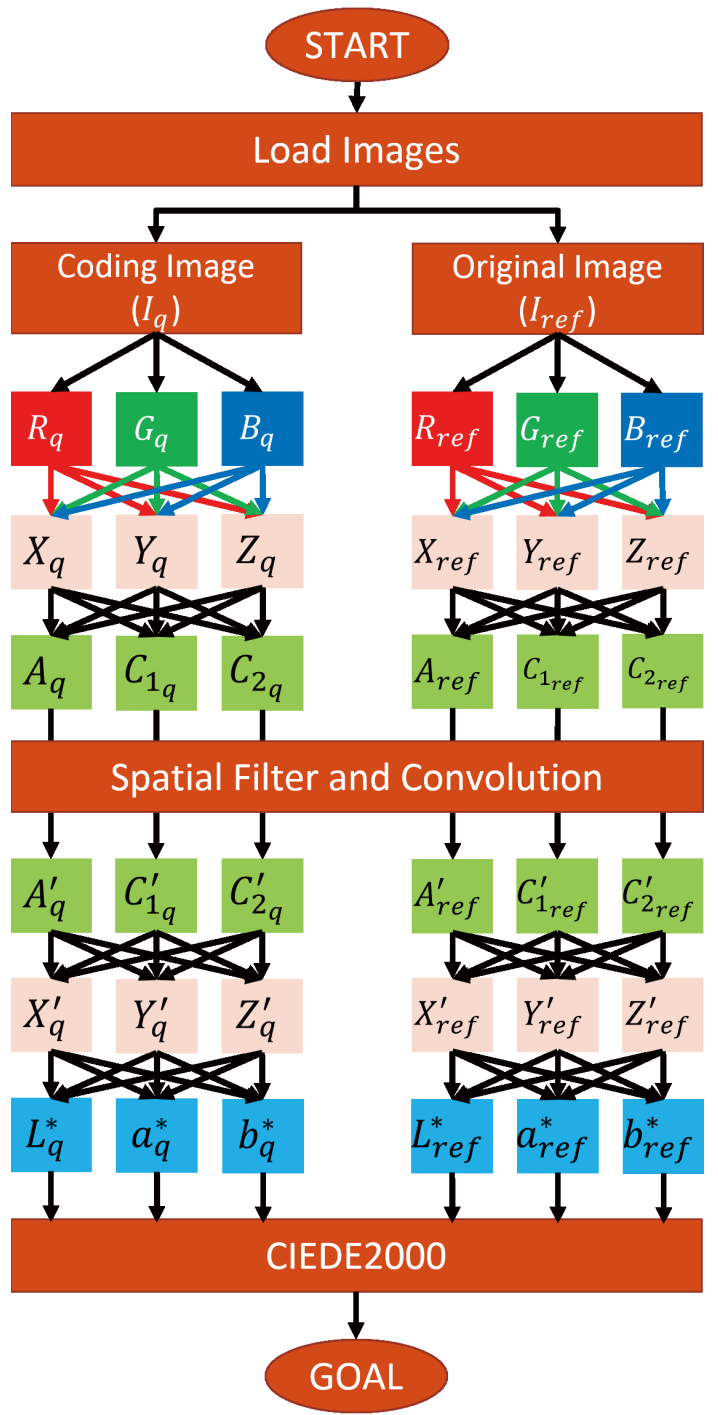

Fig. 5 S-CIELAB color space and CIEDE2000

$$
a^{*}=\left\{\begin{array}{l}
500\left[\left(\frac{X}{X_{n}}\right)^{\frac{1}{3}}-\left(\frac{Y}{Y_{n}}\right)^{\frac{1}{3}}\right] \\
\left(\frac{X}{X_{n}}, \frac{Y}{Y_{n}}>0.008856\right) \\
500 *\left[\left[7.787\left(\frac{X}{X_{n}}\right)+\frac{16}{116}\right]-\left[7.787\left(\frac{Y}{Y_{n}}\right)+\frac{16}{116}\right]\right] \\
\text { (otherwise) }
\end{array}\right.
$$$$
b^{*}=\left\{\begin{array}{l}
200\left[\left(\frac{Y}{Y_{n}}\right)^{\frac{1}{3}}-\left(\frac{Z}{Z_{n}}\right)^{\frac{1}{3}}\right] \\
\left(\frac{Y}{Y_{n}}, \frac{Z}{Z_{n}}>0.008856\right) \\
200 *\left[\left[7.787\left(\frac{Y}{Y_{n}}\right)+\frac{16}{116}\right]-\left[7.787\left(\frac{Z}{Z_{n}}\right)+\frac{16}{116}\right]\right] \\
\text { (otherwise) }
\end{array}\right.
$$

Finally, $\Delta E_{00}$ is shown in Eq. (4), (5).

$$
\Delta E_{00}=\sqrt{L^{2}+C^{2}+H^{2}+\left(R_{T} C H\right)}
$$

\begin{tabular}{|c|c|c|c|}
\hline Type of display & \multicolumn{3}{|c|}{ Newsight 3D Display 24V type } \\
\hline Display resolution & \multicolumn{3}{|c|}{ 1920×1080 (Full HD) (pixel) } \\
\hline $\begin{array}{l}\text { Display resolution } \\
\text { in the case of } \\
\text { presentation }\end{array}$ & \multicolumn{3}{|c|}{$1920 \times 960$ (pixel) } \\
\hline $\begin{array}{c}\text { Types of } \\
\text { 3D CG images }\end{array}$ & \multicolumn{3}{|c|}{ "Museum” (M), “WonderWorld" (W) } \\
\hline Types of image & \multicolumn{3}{|c|}{ Windows bitmap } \\
\hline $\begin{array}{l}\text { Encoding and } \\
\text { decoding }\end{array}$ & \multicolumn{3}{|c|}{ H.265/HEVC } \\
\hline Quantization & Exp. 1 & \multicolumn{2}{|c|}{$Q P=0$ (ref), $20,25,30,35,40,51$} \\
\hline Parameter $(Q P)$ & Exp. 2,3 & \multicolumn{2}{|c|}{$Q P=0(\mathrm{ref}), 20,30,40,51$} \\
\hline 3D system & \multicolumn{3}{|c|}{ Parallax Barrier Method } \\
\hline $\begin{array}{l}\text { The number of } \\
\text { viewpoints }\end{array}$ & \multicolumn{3}{|c|}{ Only 8 viewpoints } \\
\hline Visual range & \multicolumn{3}{|c|}{$3 \mathrm{H}$ (Height of an image) } \\
\hline Indoor lighting & \multicolumn{3}{|c|}{ None (same as dark room) } \\
\hline $\begin{array}{l}\text { Assessors' } \\
\text { position }\end{array}$ & \multicolumn{3}{|c|}{ Within horizontally $\pm 30^{\circ}$ from the center of the screen } \\
\hline \multirow{4}{*}{$\begin{array}{l}\text { Evaluation } \\
\text { experiment }\end{array}$} & $\begin{array}{l}\text { Presentation } \\
\text { time }\end{array}$ & \multicolumn{2}{|c|}{10 seconds/Contents } \\
\hline & $\begin{array}{c}\text { Evaluation } \\
\text { method }\end{array}$ & \multicolumn{2}{|c|}{$\begin{array}{l}\text { Double Stimulus Impairment Scale } \\
\text { (DSIS) }\end{array}$} \\
\hline & \multirow{2}{*}{ Assessor } & Exp. 1 & $\begin{array}{c}\text { Repeating } 6 \text { times } \\
\text { by an assessor } \\
\text { (Graduate school student) }\end{array}$ \\
\hline & & Exp. 2, 3 & $\begin{array}{c}\text { Repeating } 5 \text { times } \\
\text { by an assessor } \\
\text { (Graduate school student) }\end{array}$ \\
\hline
\end{tabular}

Table 1 Main specifications of experiments in this study

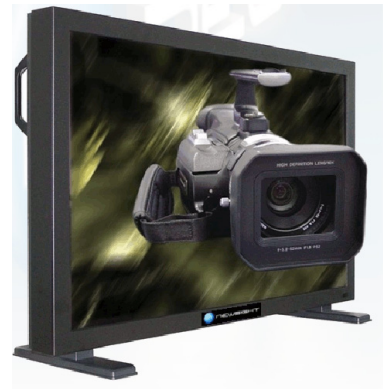

Fig. 6 Appearance of 3D display. [17]

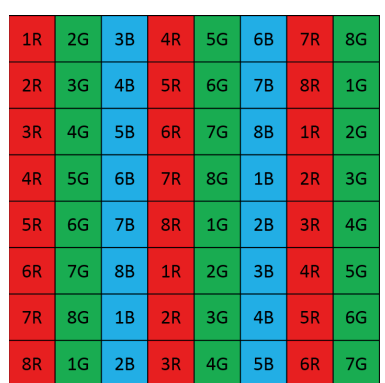

Fig. 7 Pixels and viewpoint arrangement of 3D display.

$$
L=\frac{\Delta L^{\prime}}{K_{L} \cdot S_{L}}, C=\frac{\Delta C^{\prime}}{K_{C} \cdot S_{C}}, H=\frac{\Delta H^{\prime}}{K_{H} \cdot S_{H}}
$$

\subsection{Experimental Contents}

Table 1 lists the experimental conditions and specifications. The experimental environment was based on the ITU-R BT.500-13 recommendation [15]. We used a parallax barrier display, as shown in Fig. 6. Figure 7 shows the pixel arrangement in the parallax barrier display. In the pixel arrangement, each viewpoint is arranged consistently as $\mathrm{R}, \mathrm{G}$, and $\mathrm{B}$, in the direction of the right diagonal. In the $3 \mathrm{D}$ display mechanism, images can be seen in the $3 \mathrm{D}$ display from different fields of view by dividing $R, G$, and $B$ through the parallax barrier in front of an LCD display. When we displayed a 3D image on the 3D display, we performed a realtime parallax mix automatically by using a media player provided by Newsight Corporation [17]. Further, on the basis of the experimental results reported in [12], we defined 


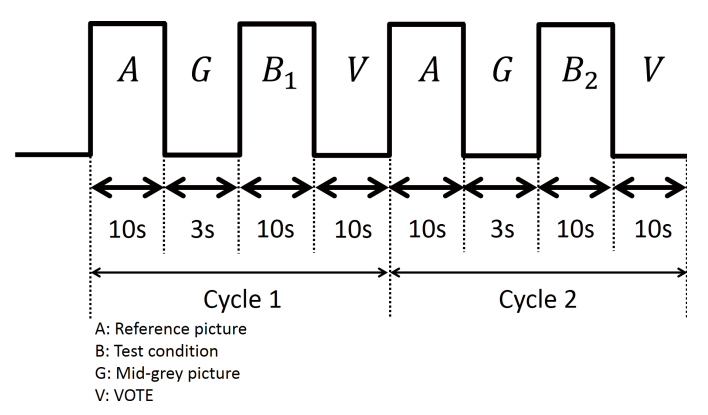

Fig. 8 Double Stimulus Impairment Scale (DSIS)

Table 2 EBU quality scale of experiments

\begin{tabular}{c|c}
\hline Score & Quality \\
\hline \hline 5 & Imperceptible \\
\hline 4 & Perceptible, but not annoying \\
\hline 3 & Slightly Annoying \\
\hline 2 & Annoying \\
\hline 1 & Very Annoying \\
\hline
\end{tabular}

the CG camera interval.

\subsection{Method of Experiment and Evaluation}

Figure 8 shows the presentation order and image sequence time in the experiment (double stimulus impairment scale: DSIS). First, we displayed reference image A for $10 \mathrm{~s}$ and then, the mid-gray image $\mathrm{G}$ for $3 \mathrm{~s}$. Then, we displayed the test condition image B for $10 \mathrm{~s}$. Next, the assessor evaluated this cycle and input the evaluation value into the computer application (VOTE), which took $10 \mathrm{~s}$. This cycle was defined as one set, and we repeated the cycle until the last set. When the assessors performed the subjective evaluation for more than $30 \mathrm{~min}$, they suffered from accumulated fatigue (such as visual fatigue) [18]. Therefore, when the subjective evaluation time was more than 30 min, we divided the assessors' subjective evaluation time into intervals of several seconds each. In this experiment, the assessors input the evaluation scores by using a computer application. Table 2 shows the evaluation standard (European Broadcasting Union: EBU method) [15]. The assessors gave the evaluation scores according to five ranks (mean opinion score: MOS (Museum: $M_{M O S}$, Wonder World : $\left.W_{M O S}\right)$ ). Here, we defined $M O S=4.5,3.5,2.5$ as the "detective limit (DL)," "acceptability limit (AL)," and "endurance limit (EL)," respectively. In this study, we each assessor repeated the evaluation six times (Exp. 1) or five times (Exps. 2 and 3). The image sequences were displayed randomly in each experiment. We included a rest time of $30 \mathrm{~min}$. Thus, we could reach an appropriate conclusion.

\section{Result of Exp. 1 and Discussion}

\subsection{Result of Exp. 1-1}

Figure 9 shows the result of the subjective evaluation experiment Exp. 1-1. The vertical axis represents $M O S$, and the

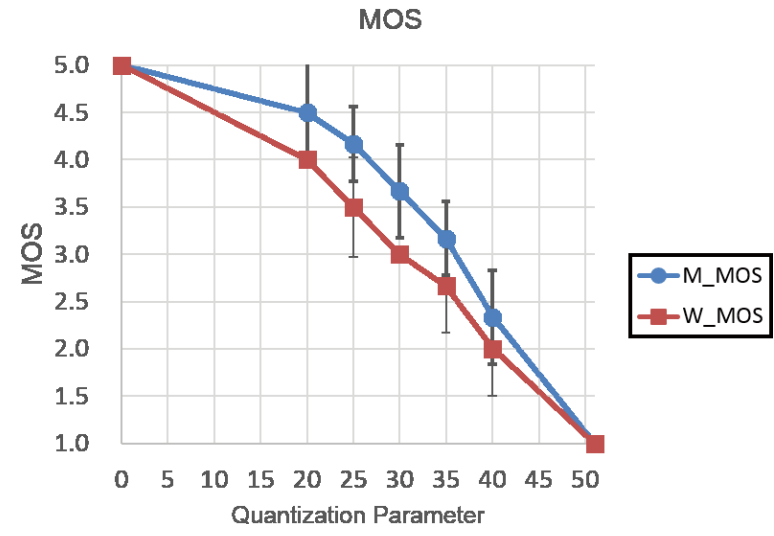

Fig.9 Result of Exp. 1-1

horizontal axis denotes $Q P$. The error bar is extended up and down from the plot points in Fig. 9, which shows a 95\% confidence interval.

\subsubsection{Experimental Result of Subjective Evaluation}

From the result of Exp. 1-1, we infer that the higher the $Q P$ value is, the lower are $M_{M O S}$ and $W_{M O S}$. Thus, $M_{M O S} \geq$ $W_{M O S}$ is satisfied. $M_{M O S}$ tends to "DL" in the case of $Q P \leq$ 20 , tends to "AL" in the case of $20<Q P<35$, and tends to "EL" in the case of $Q P \geq 35$. When $Q P \geq 35$ is satisfied, we can see that $M_{M O S}$ and $W_{M O S}$ tend to decreased dramatically and satisfy "EL."

\subsubsection{Statistical Learning by SVM}

In the last subsection, we showed results by MOS, however, we did not find out whether it is possible for sequence patterns to classify or not. Therefore, we considered that we need to classify them statistically by using a SVM. SVM is a method that evaluates the decision boundary when the margin of the learning data is the maximum [10], [19]. The expectancy of SVM is as follows:

$$
y(\mathrm{x})=\sum_{n=1}^{N} a_{n} t_{n} k\left(\mathrm{x}, \mathrm{x}_{n}\right)+b
$$

Equation (6) shows the weighting addition of the kernel function $k$ with the support vector (input vector $x$ ) and the bias term $b$. Table 3 shows SVM (sequential minimal optimization (SMO)) algorithm provided in Weka 3.6 [20][22]. The number of data samples is the same as the number of trials for the evaluation. SVM used in this study, which is used the kernel function as shown in Eq. (6), and SVM class based on $Q P$. The "Precision," "Recall," and "FMeasure" values greater than 0.7 are denoted in boldface font.

From the results of SVM $(Q P)$, we infer that in classes "QP_ref," "QP_40," and "QP_51," all parameter values are more than 0.7. Therefore, we can judge acceptable. In class "QP_20," the "Recall" and "F-Measure" values are more 
Table $3 \quad \operatorname{SVM}(Q P)$ in Exp. 1

\begin{tabular}{|c|c|c|c|}
\hline Precision & Recall & F-Measure & Class $(Q P)$ \\
\hline \hline $\mathbf{1}$ & $\mathbf{1}$ & $\mathbf{1}$ & QP_ref \\
\hline 0.67 & $\mathbf{1}$ & $\mathbf{0 . 8 0}$ & QP_20 \\
\hline 0 & 0 & 0 & QP_25 \\
\hline 0.50 & 0.67 & 0.57 & QP_30 \\
\hline 0.60 & 0.50 & 0.55 & QP_35 \\
\hline $\mathbf{0 . 7 5}$ & $\mathbf{1}$ & $\mathbf{0 . 8 6}$ & QP_40 \\
\hline $\mathbf{1}$ & $\mathbf{1}$ & $\mathbf{1}$ & QP_51 \\
\hline 0.65 & 0.74 & 0.68 & Weighted Avg. \\
\hline
\end{tabular}

Table 4 SVM correctly classification percentage (Exp. 1)

\begin{tabular}{|c|c|c|c|c|c|}
\hline Class & $\begin{array}{c}\text { Total } \\
\text { Number } \\
\text { of Instances }\end{array}$ & $\begin{array}{c}\text { Correctly } \\
\text { Classified } \\
\text { Instances }\end{array}$ & Percentage & $\begin{array}{c}\text { Mean } \\
\text { absolute } \\
\text { error }\end{array}$ & $\begin{array}{c}\text { Root mean } \\
\text { squared } \\
\text { error }\end{array}$ \\
\hline $\begin{array}{c}\text { Exp. 1 } \\
\text { ("QP") }\end{array}$ & 42 & 31 & $73.8 \%$ & 0.21 & 0.31 \\
\hline
\end{tabular}

than 0.7. Therefore, we can judge slightly acceptable. On the other hand, in classes "QP_25," "QP_30," and "QP_35," all parameter values are not more than 0.7. Therefore, we can judge not acceptable. On the whole, we can see that the parameter values are more than 0.7 when $Q P$ is higher or lower. Thus, we consider that it is easy for the assessor to perceive the coded degradation when $Q P$ is higher or lower.

Table 4 shows the correctly classified percentage of $(Q P)$. From Table 4, we find that in class " $Q P$," the SVM's correct classification percentage is $73.8 \%$ (31/42), which is higher than $70 \%$. Therefore, we can judge "classified" in class " $Q P . "$

\subsection{Result of Exp. 1-2}

Figure 10 shows the result of Exp. 1-2 ("Museum" and "Wonder World"). The vertical axis represents $L^{*}$ (of $\left.L^{*} a^{*} b^{*}\right)$, and the horizontal axis denotes $Q P$. In Fig. 10, $L^{*}$ of "Museum" (After this, $L_{m}^{*}$ ) and $L^{*}$ of "Wonder World" (After this, $L_{w}^{*}$ ), which are the luminance in each images, and they are generated summation of the luminance values for the image pixels by eight viewpoints.

From Fig. 10, we can see that $L_{m}^{*}$ is between 76 and 78 for all $Q P$ patterns. On the other hand, $L_{w}^{*}$ is before and after 24. Thus, in Exp. 1, we can see that $L_{m}^{*}$ is higher than $L_{w}^{*}$ in terms of luminance.

\subsection{Result of Exp. 1-3}

Figure 11 shows the result of Exp. 1-3 ("Museum" and "Wonder World"). The vertical axis denotes $\Delta E_{00}$ (CIEDE2000 color difference), and the horizontal axis represents $Q P$. In Fig. 11, $\Delta E_{00}$ of "Museum" (hereafter, $\Delta E_{00 m}$ ) and $\Delta E_{00}$ of "Wonder World" (hereafter, $\Delta E_{00 w}$ ), which are the color difference in each image, are generated by a summation of the color difference values for the image pixels from the eight viewpoints.

From Fig. 11, we can infer that the higher the $Q P$ is, the higher are both $\Delta E_{00 m}$ and $\Delta E_{00 w}$. When $Q P>30$ is satisfied, $\Delta E_{00 w}$ tends to increase dramatically. On the other
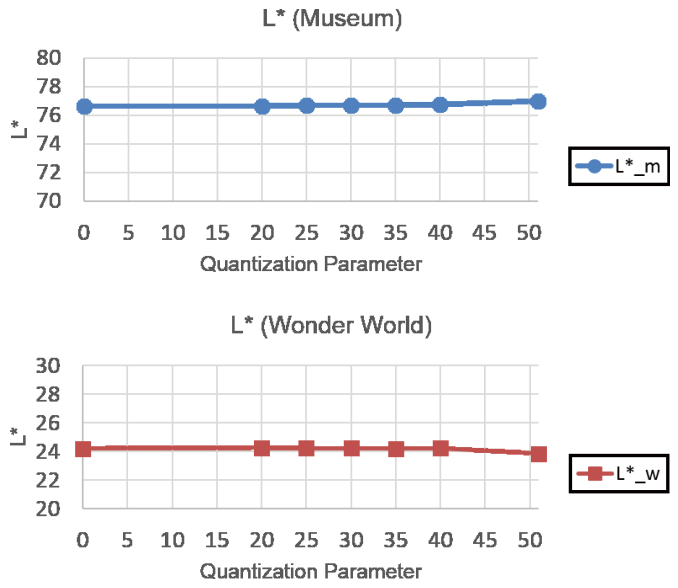

Fig. 10 Result of Exp. 1-2

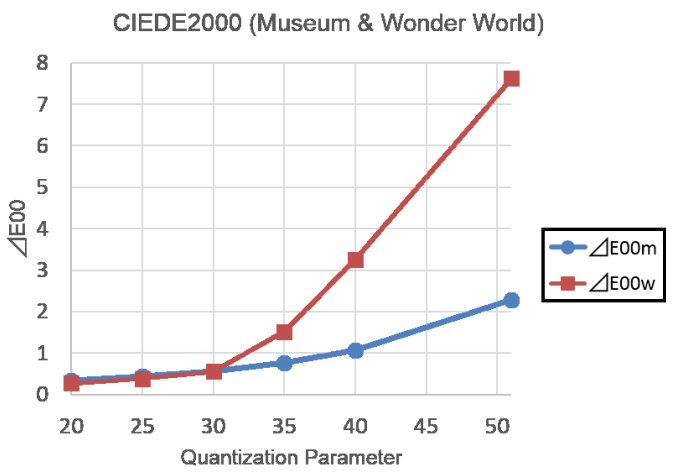

Fig. 11 Result of Exp. 1-3

hand, $\Delta E_{00 m}$ tends to increase gradually when $Q P>30$ is satisfied. As a result, compared to Exp. 1-1, we can see as if Fig. 11 drew the parabolic orbit, contrary to Fig. 9.

\subsection{Discussion}

From the result of Exp. 1, we infer that when $Q P \geq 35$ is satisfied, $M_{M O S}$ and $W_{M O S}$ tend to decrease dramatically. On the other hand, when $Q P \geq 30$ is satisfied, $\Delta E_{00 m}$ and $\Delta E_{00 w}$ tend start increasing gradually. Thus, we believe that the coded degradation obtained by changing the QP of H.265/HEVC and the CIEDE2000 color difference in a multi-view 3D image are related. On the other hand, with respect to the luminance, we can see the difference in the luminance between the 3D image contents "Museum" and "Wonder World." However, when we changed the $Q P$ of the $3 \mathrm{D}$ image, we could not see the change in luminance. From this, we cannot see the change for the luminance, although we changed the quantization parameter of the 3D image in the case of occurring no luminance change. However, we consider that we may obtain something to knowledge in the case of occurring the luminance change in Exp. 2 and 3. 


\section{Result of Exp. 2 and Discussion}

\subsection{Result of Exp. 2-1}

Figures 12-16 show the results of Exp. 2-1. The vertical axis represents $M O S$, and the horizontal axis denotes $Q P$, as in the case of Exp. 1-1. The error bar is extended up and down from the plot points in Figs. 12-16, which shows a $95 \%$ confidence interval.

\subsubsection{Experimental Result of Subjective Evaluation}

From the result of Exp. 2-1, we can infer that satisfaction

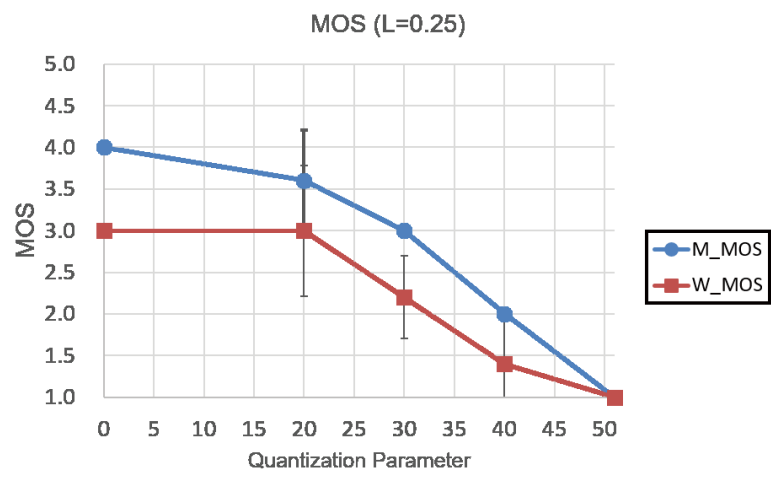

Fig. 12 Result of Exp. 2-1 $(L=0.25)$

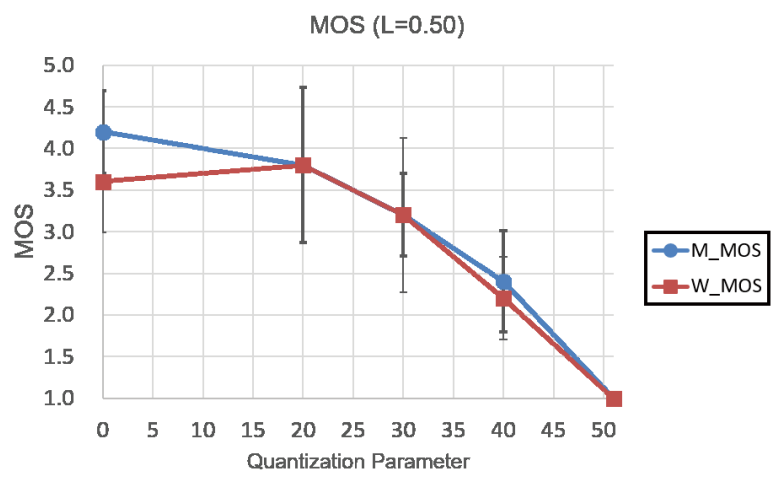

Fig. 13 Result of Exp. 2-1 $(L=0.50)$

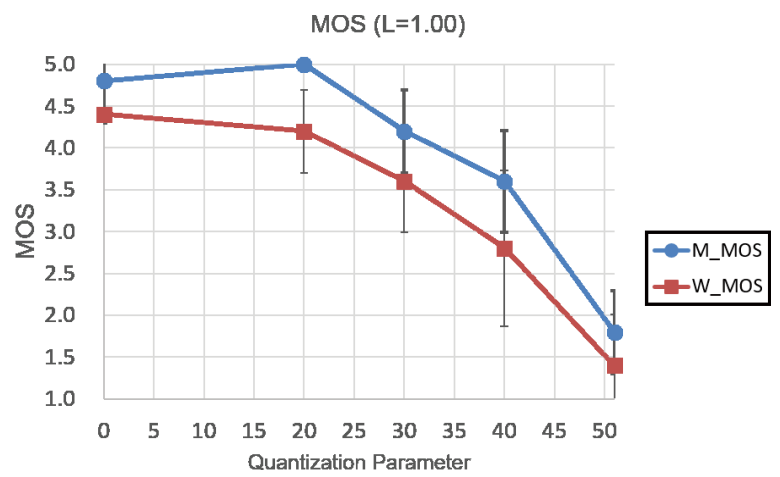

Fig. 14 Result of Exp. 2-1 $(L=1.00)$ than "DL" in ref $\leq Q P \leq 40$ in case $L$ equals 1.00 and 2.00. Similarly, we can observe that $L=1.00,2.00, L=0.50$, and $L=0.25,4.00$. In the case of $L=1.00,2.00$, when $Q P \leq 20$ is satisfied, $M_{M O S}$ satisfies "DL" and $W_{M O S}$ satisfies "AL." This group has the maximum $M O S$ value among all groups. In the case of $L=0.50$, we can see that $M_{M O S}$ equals $W_{M O S}$ in almost all cases except for $Q P=r e f$. This tendency is only observed when $L=0.50$, and $M_{M O S}$ and $W_{M O S}$ are changed regularly. In the case of $L=0.25,4.00$, we can see that both $M_{M O S}$ and $W_{M O S}$ are not greater than $M O S=4$. This result shows the worst tendency of all groups. In the case of $L=0.25$, the image contrast is comparatively high. On the other hand, in the case of $L=4.00$, the image contrast is comparatively bright. Comparing both the patterns, we find that the high-contrast image $(L=0.25)$ declines gradually in the case of $Q P=30,40$; however, the brightcontrast image $(L=4.00)$ remains unchanged (flat) in the case of $Q P=30,40$. Thus, we conclude that it is easy not for the bright-contrast image but for the high-contrast image to perceive the coded degradation by using H.265/HEVC.

\subsubsection{Statistical Learning by SVM}

Tables 5 and 6 show the SVM (class Lum, $Q P$ ) provided in Weka 3.6, as in the case of Exp. 1. The "Precision," "Recall," and "F-Measure" values greater than 0.7 are denoted in boldface font, as in the case of Exp. 1. From the result of SVM (Lum), we can infer that the "Recall" value is greater

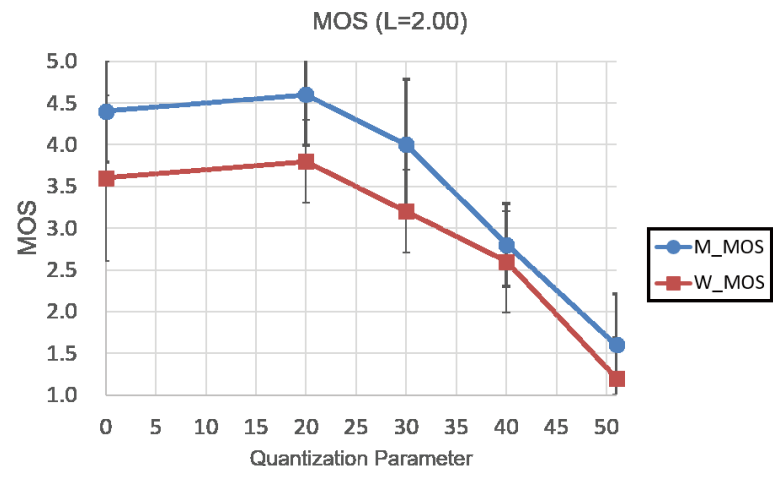

Fig. 15 Result of Exp. 2-1 $(L=2.00)$

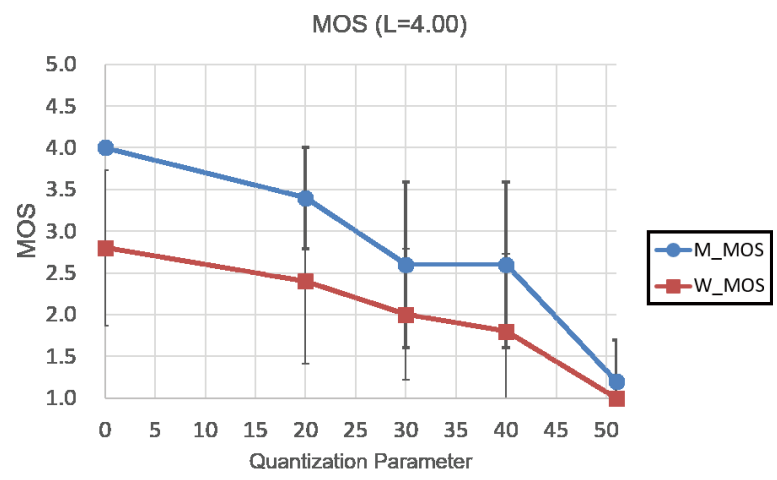

Fig. 16 Result of Exp. 2-1 $(L=4.00)$ 
Table 5 SVM (Lum) in Exp. 2

\begin{tabular}{|c|c|c|c|}
\hline Precision & Recall & F-Measure & Class (Lum) \\
\hline \hline 0.33 & 0.20 & 0.25 & Lum_025 \\
\hline 0.36 & 0.32 & 0.34 & Lum_050 \\
\hline 0.45 & $\mathbf{0 . 7 6}$ & 0.57 & Lum_1 \\
\hline 0.40 & 0.16 & 0.23 & Lum_2 \\
\hline 0.37 & 0.60 & 0.46 & Lum_4 \\
\hline 0.37 & 0.39 & 0.35 & Weighted Avg. \\
\hline
\end{tabular}

Table 6 SVM $(Q P)$ in Exp. 2

\begin{tabular}{|c|c|c|c|}
\hline Precision & Recall & F-Measure & Class $(Q P)$ \\
\hline \hline 0.55 & $\mathbf{0 . 8 7}$ & 0.68 & QP_ref \\
\hline 0.50 & 0.04 & 0.07 & QP_20 \\
\hline 0.44 & 0.68 & 0.53 & QP_30 \\
\hline $\mathbf{0 . 7 0}$ & 0.28 & 0.40 & QP_40 \\
\hline $\mathbf{0 . 7 8}$ & $\mathbf{1}$ & $\mathbf{0 . 8 8}$ & QP_51 \\
\hline 0.59 & 0.59 & 0.52 & Weighted Avg. \\
\hline
\end{tabular}

Table $7 \quad$ SVM correctly classification percentage (Exp. 2)

\begin{tabular}{|c|c|c|c|c|c|}
\hline Class & $\begin{array}{c}\text { Total } \\
\text { Number } \\
\text { of Instances }\end{array}$ & $\begin{array}{c}\text { Correctly } \\
\text { Classified } \\
\text { Instances }\end{array}$ & Percentage & $\begin{array}{c}\text { Mean } \\
\text { absolute } \\
\text { error }\end{array}$ & $\begin{array}{c}\text { Root mean } \\
\text { squared } \\
\text { error }\end{array}$ \\
\hline $\begin{array}{c}\text { Exp. 2 } \\
\text { ("Lum") }\end{array}$ & 130 & 51 & $39.2 \%$ & 0.25 & 0.35 \\
\hline $\begin{array}{c}\text { Exp. 2 } \\
\text { ("QP") }\end{array}$ & 130 & 76 & $58.4 \%$ & 0.26 & 0.35 \\
\hline
\end{tabular}

than 0.7 in "Lum1." The other patterns are lower than 0.7. From the result of SVM $(Q P)$, we can infer that the "Precision" value is greater than 0.7 in "QP_40" and "QP_51." Further, the "Recall" value is greater than 0.7 in "QP_ref" and "QP_51," and the "F-Measure" value is greater than 0.7 in "QP_51." In "QP_51," we can see the satisfaction of all items. By comparing SVM (Lum) to SVM $(Q P)$, in terms of the satisfaction, we can infer that the value of SVM $(Q P)$ is better than that of SVM (Lum).

Table 7 shows the SVM's correctly classified percentage of (Lum, $Q P)$. From Table 7, we find that in class "Lum," the SVM's correct classification percentage is $39.2 \%$ (51/130). Therefore, we can judge "not classified." In class " $Q P$," the SVM's correct classification percentage is $58.4 \%$ (76/130). Therefore, we can judge "not bad classified."

\subsection{Result of Exp. 2-2}

Figures 17 and 18 show the result of Exp. 2-2 ("Museum" and "Wonder World"). The vertical axis represents $L^{*}$ (of $\left.L^{*} a^{*} b^{*}\right)$, and the horizontal axis denotes the $Q P$, as in the case of Exp. 1-2. In Figs. 17 and 18, $L_{m}^{*}$ and $L_{w}^{*}$ represent the luminance in each image and are generated by a summation of the luminance values for the image pixels for the eight viewpoints, as in the case of Exp. 1-2.

From Figs. 17 and 18, we can infer that $L_{m}^{*}$ is higher than $L_{w}^{*}$ in all $Q P$ patterns, as in Exp. 1-2. In Exp. 2, we changed the luminance of an image by using contrast enhancement. For $L^{*}$, we can see that the difference between " $L^{*}$ _lum 0.50 " and " $L^{*}$ _lum 1.00 " is the maximum among all luminance patterns for $L_{m}^{*}$. However, for $L_{w}^{*}$, we cannot observe this difference. Thus, we believe that it is easy not in

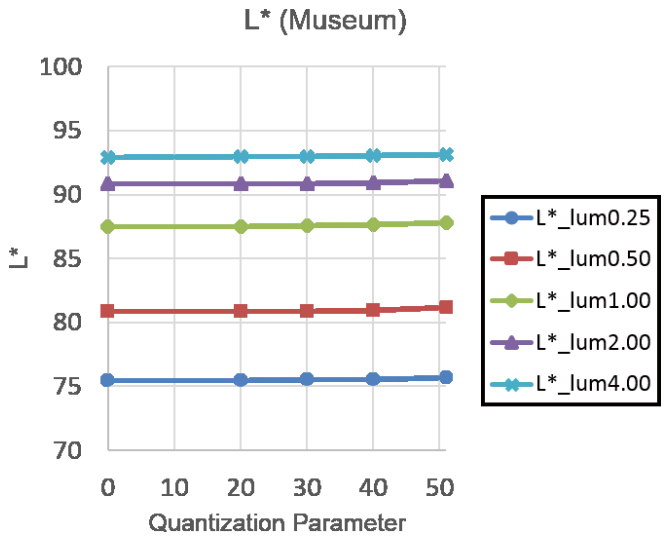

Fig. 17 Result of Exp. 2-2 (Museum)

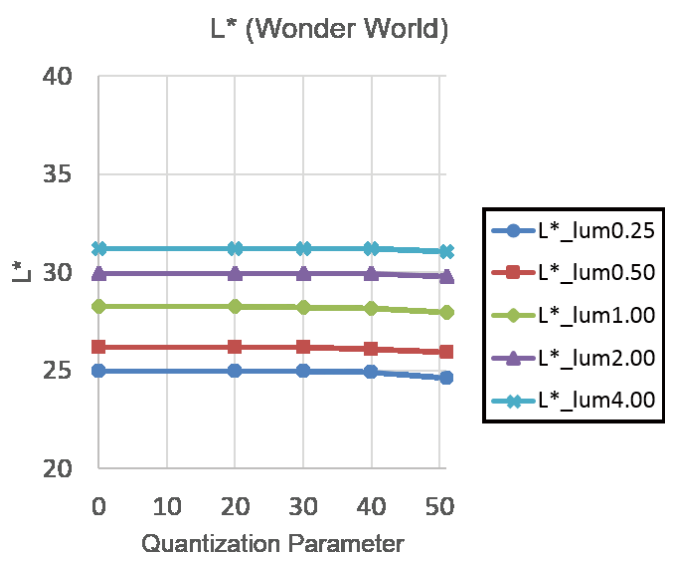

Fig. 18 Result of Exp. 2-2 (Wonder World)

the case of "Wonder World" but in the case of "Museum" to change $L^{*}$. This can be seen from the range between the maximum and the minimum $L^{*}$. The range of $L_{m}^{*}$ is $75 \leq L^{*} \leq 93$. The range of $L_{w}^{*}$ is $25 \leq L^{*} \leq 32$. For the $Q P$ change, we cannot see the characterization, as in the case of Exp. 1-2.

\subsection{Result of Exp. 2-3}

Figures 19 and 20 show the result of Exp. 2-3 ("Museum" and "Wonder World"). The vertical axis represents $\Delta E_{00}$ (CIEDE2000 color difference), and the horizontal axis denotes the $Q P$, as in the case of Exp. 1-3. In Figs. 19 and 20, $\Delta E_{00 m}$ and $\Delta E_{00 w}$ denote the color difference in each image and are generated by a summation of the color difference values for the image pixels for the eight viewpoints, as in the case of Exp. 1-3.

From Figs. 19 and 20, we can infer that $\Delta E_{00 w}$ is higher than $\Delta E_{00 m}$ in all $Q P$ patterns. However, the range of $\Delta E_{00 w}$ is larger than that of $\Delta E_{00 m}$. Comparing Exp. 2-3 to Exp. 1-3, we find that in the case of Exp. 2-3, we cannot observe a characteristic change, as that in the case of Exp. 1-3. Further, by comparing Exp. 2-3 to Exp. 2-2, we can observe the inverse tendency. In the case of Exp. 2-3, we can observe that $L_{m}^{*}$ is higher than $L_{w}^{*}$. On the other hand, we can observe 


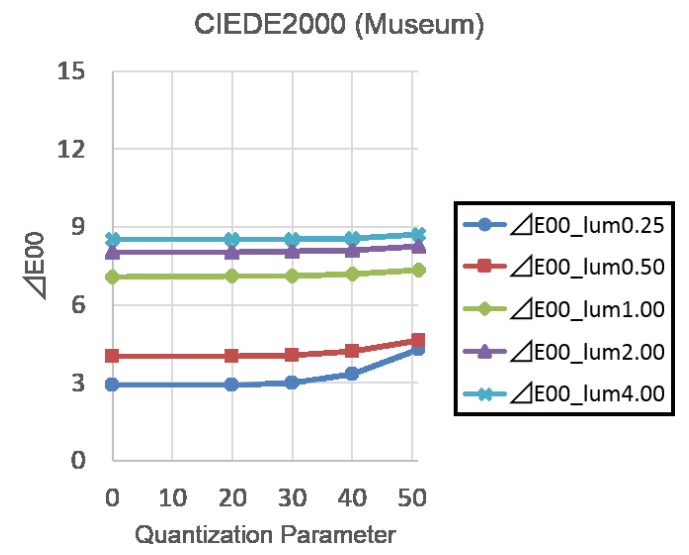

Fig. 19 Result of Exp. 2-3 (Museum)

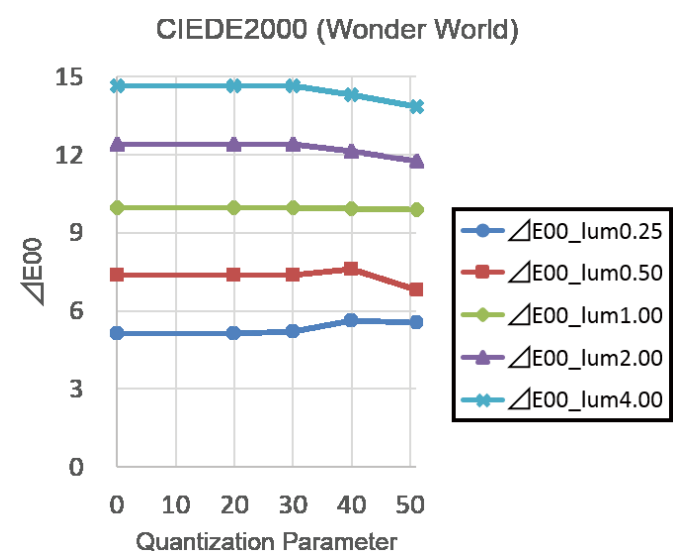

Fig. 20 Result of Exp. 2-3 (Wonder World)

that $\Delta E_{00 w}$ is higher than $\Delta E_{00 m}$. Thus, we believe that there is a trade-off relationship between $L^{*}$ and $\Delta E_{00}$.

\subsection{Discussion}

From the result of Exp. 2, we infer that the luminance of "Museum" is greater than that of "Wonder World"; however, the difference in CIEDE2000 in the case of "Museum" is lower than that in the case of "Wonder World." Further, from the results of Exp. 2-1, we can infer that $M_{M O S}$ is higher than $W_{M O S}$. Thus, we conclude that the luminance change gives the affection to assessment for the image contents. From the results of SVM, we infer that SVM $(Q P)$ has a higher value than SVM (Lum). We believe that even when the luminance change is little, an assessor does not perceive the image quality and the degradation, nevertheless experimenting several image quality assessment tests.

\section{Result of Exp. 3 and Discussion}

\subsection{Result of Exp. 3-1}

Figures 21-25 show the result of Exp. 3-1. The vertical axis denotes $M O S$, and the horizontal axis represents $Q P$, as in the case of Exp. 2-1. The error bar is extended up

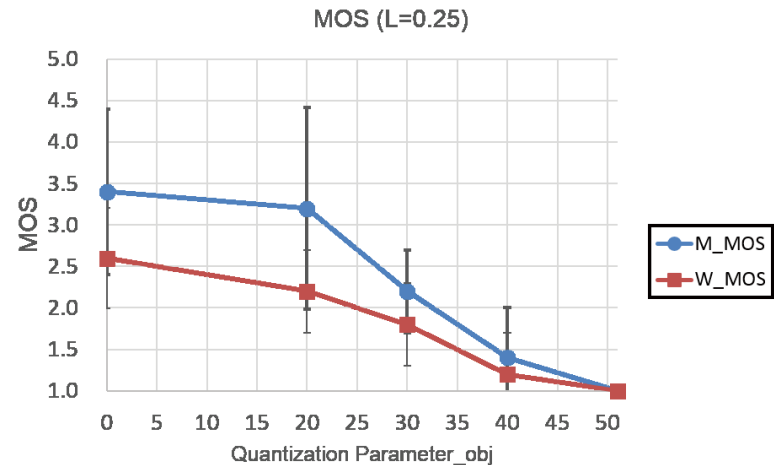

Fig. 21 Result of Exp. 3-1 $(L=0.25)$

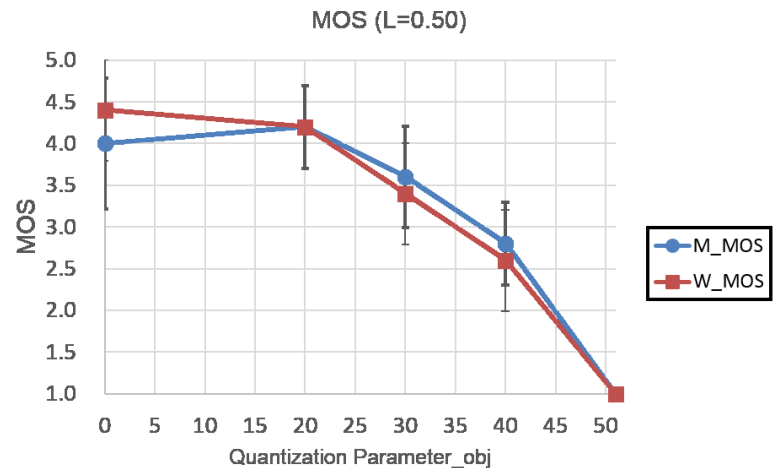

Fig. 22 Result of Exp. 3-1 $(L=0.50)$

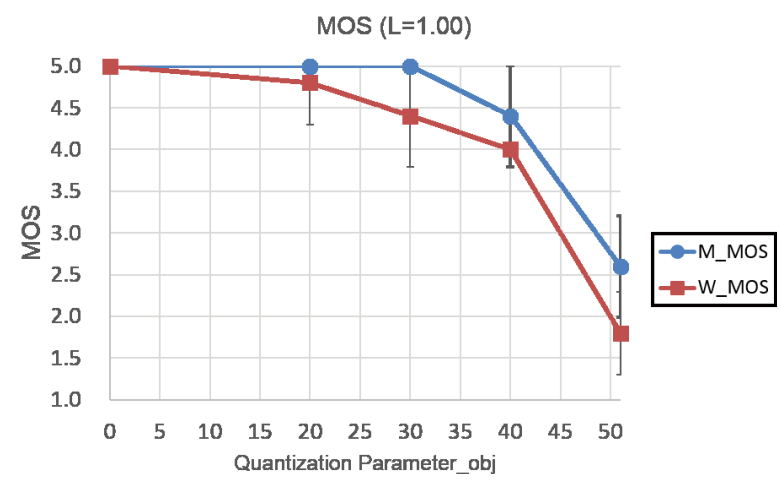

Fig. 23 Result of Exp. 3-1 $(L=1.00)$

and down from the plot points in Figs. 21-25, which shows a $95 \%$ confidence interval.

\subsubsection{Experimental Result of Subjective Evaluation}

From the result of Exp. 3, in the case of $L=0.25$, we can infer that $M_{M O S}$ is greater than "EL" when $Q P_{o b j}<30$; however, $W_{M O S}$ is lower than "EL" in all patterns. With respect to the error bar, $M_{M O S}$ and $W_{M O S}$ tend to increase when $Q P_{o b j}=0(r e f), 20$. In the case of $L=0.50, M_{M O S}$ and $W_{M O S}$ are greater than "AL" when $Q P_{o b j} \leq 30$, and are greater than "EL" when $Q P_{o b j} \leq 40$. In the case of $L=1.00, M_{M O S}$ is greater than "DL" when $Q P_{o b j} \leq 40$, and $W_{M O S}$ is greater than "DL" when $Q P_{o b j} \leq 30$. In the case 


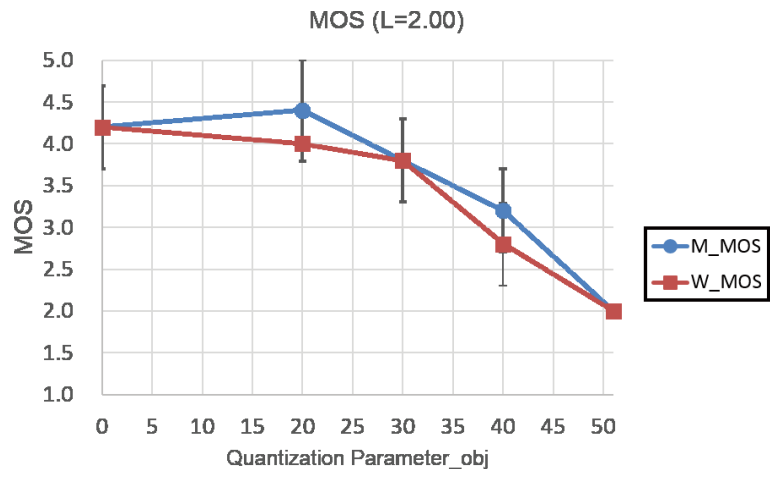

Fig. 24 Result of Exp. 3-1 $(L=2.00)$

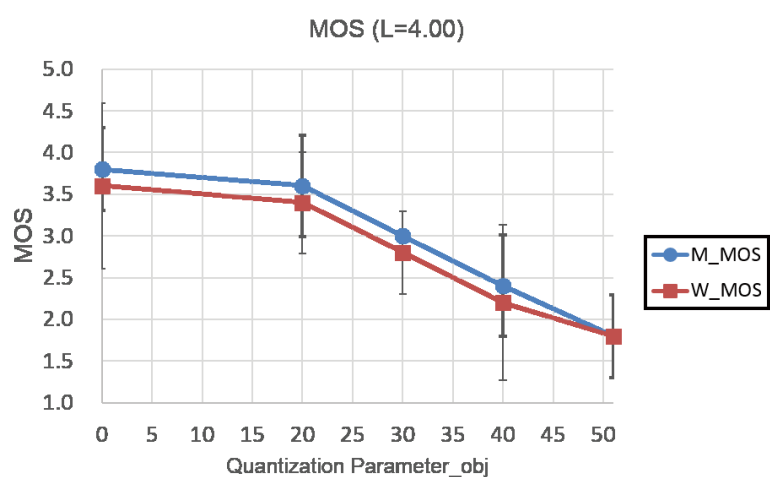

Fig. 25 Result of Exp. 3-1 $(L=4.00)$

of $L=1.00$, we can see the best evaluation values of all. In the case of $L=2.00$, we can see the difference between $M_{M O S}$ and $W_{M O S}\left(M_{M O S}>W_{M O S}\right)$ when $Q P_{o b j}=20,40$. Furthermore, $M_{M O S}$ and $W_{M O S}$ are greater than "AL" when $Q P_{o b j} \leq 30$, and are greater than "EL" when $Q P_{o b j} \leq 40$. In the case of $L=4.00, M_{M O S}$ is greater than $W_{M O S}$ when $Q P_{o b j} \leq 40$; however, we can see some similarity in the graph shape. On the whole, we can observe the steadiness of the evaluation in that the difference among the $M O S$ patterns with a high contrast $(L=0.25,0.50)$ is more than the difference among $M O S$ patterns without a high contrast $(L=2.00,4.00)$.

\subsubsection{Statistical Learning by SVM}

Tables 8 and 9 show the SVM (class Lum, $Q P$ ) provided in Weka 3.6, as in the cases of Exps. 1 and 2. The "Precision," "Recall," and "F-Measure" values greater than 0.7 are denoted in boldface font, as in the cases of Exps. 1 and 2. From the result of SVM ( $L u m)$, we find that with respect to "Precision," only class "Lum_05" has a value greater than 0.7. In terms of "Recall," classes "Lum_0.25" and "Lum_1" have values of more than 0.7. Further, in terms of "F-Measure," classes "Lum_0.25" and "Lum_1" have values of more than 0.7. "Lum_2" and "Lum_4" are few patterns more than 0.7 ; therefore, we consider that satisfy in case the image contrast is deep. From the result of $\operatorname{SVM}(Q P)$, we infer that with respect to "Precision," classes
Table 8 SVM (Lum) in Exp. 3

\begin{tabular}{|c|c|c|c|}
\hline Precision & Recall & F-Measure & Class (Lum) \\
\hline \hline 0.67 & $\mathbf{0 . 8 8}$ & $\mathbf{0 . 7 6}$ & Lum_025 \\
\hline $\mathbf{1}$ & 0.04 & 0.08 & Lum_050 \\
\hline 0.65 & $\mathbf{0 . 8 0}$ & $\mathbf{0 . 7 1}$ & Lum_1 \\
\hline 0.39 & 0.64 & 0.49 & Lum_2 \\
\hline 0.50 & 0.48 & 0.49 & Lum_4 \\
\hline 0.62 & 0.55 & 0.49 & Weighted Avg. \\
\hline
\end{tabular}

Table 9 SVM $(Q P)$ in Exp. 3

\begin{tabular}{|c|c|c|c|}
\hline Precision & Recall & F-Measure & Class $(Q P)$ \\
\hline \hline 0.46 & $\mathbf{0 . 8 7}$ & 0.61 & QP_ref \\
\hline $\mathbf{1}$ & 0.04 & 0.08 & QP_20 \\
\hline 0.49 & 0.60 & 0.54 & QP_30 \\
\hline $\mathbf{1}$ & 0.32 & 0.49 & QP_40 \\
\hline $\mathbf{0 . 7 4}$ & $\mathbf{1}$ & $\mathbf{0 . 8 5}$ & QP_51 \\
\hline 0.73 & 0.58 & 0.51 & Weighted Avg. \\
\hline
\end{tabular}

Table 10 SVM correctly classification percentage (Exp. 3)

\begin{tabular}{|c|c|c|c|c|c|}
\hline Class & $\begin{array}{c}\text { Total } \\
\text { Number } \\
\text { of Instances }\end{array}$ & $\begin{array}{c}\text { Correctly } \\
\text { Classified } \\
\text { Instances }\end{array}$ & Percentage & $\begin{array}{c}\text { Mean } \\
\text { absolute } \\
\text { error }\end{array}$ & $\begin{array}{c}\text { Root mean } \\
\text { squared } \\
\text { error }\end{array}$ \\
\hline $\begin{array}{c}\text { Exp. 3 } \\
\text { ("Lum") }\end{array}$ & 130 & 71 & $54.6 \%$ & 0.24 & 0.34 \\
\hline $\begin{array}{c}\text { Exp. 3 } \\
\text { ("QP") }\end{array}$ & 130 & 75 & $57.7 \%$ & 0.26 & 0.35 \\
\hline
\end{tabular}

"QP_20," "QP_40," and "QP_51" have values more than 0.7. In terms of "Recall," classes "QP_ref" and "QP_51" have values greater than 0.7. Further, in terms of "F-Measure," only class "QP_51" has a value of more than 0.7 . In general, class "QP_51" is more than 0.7 of all. Therefore, on the basis of the result of "QP_51," we believe that there is a relation between SVM $($ Lum $)$ and SVM $(Q P)$.

Table 10 shows the correctly classified percentage $\left(L^{*}\right.$ and $Q P)$. The percentage of class "Lum" is $54.6 \%$ (71/130). We can judge "not badly classified." On the other hand, the percentage of class " $Q P$ " is $57.7 \%$ (75/130). We can judge "not badly classified," as in the case of class "Lum."

\subsection{Result of Exp. 3-2}

Figures 26 and 27 show the result of Exp. 3-2 ("Museum" and "Wonder World"). The vertical axis represents $L^{*}$ (of the $L^{*} a^{*} b^{*}$ space), and the horizontal axis denotes $Q P$, as in the cases of Exps. 1-2 and 2-2. In Figs. 26 and 27, $L_{m}^{*}$ and $L_{w}^{*}$ denote the luminance in each image and are generated by a summation of the luminance values for the image pixels for eight viewpoints, as in the case of Exps. 1-2 and 2-2.

From Figs. 26 and 27, we infer that $L_{m}^{*}$ is higher than $L_{w}^{*}(76-78 v s .24-25)$ without $Q P_{o b j}$ patterns. In the case of luminance $L^{*}$, we can observe that the range of $L_{m}^{*}$ is larger than that of $L_{w}^{*}$. Thus, for a coded degradation of the object region, we believe that it is easy not for "Wonder World" but for "Museum" to affect $L^{*}$ by the contrast enhancement processing. On the other hand, compared to the case of Exp. 2-2, we can infer that the $L^{*}$ range of Exp. 3-2 is narrower than that of Exp. 2-2 without $Q P$ patterns. In particular, we can judge that the range of $L_{w}^{*}$ is considerably narrower in 


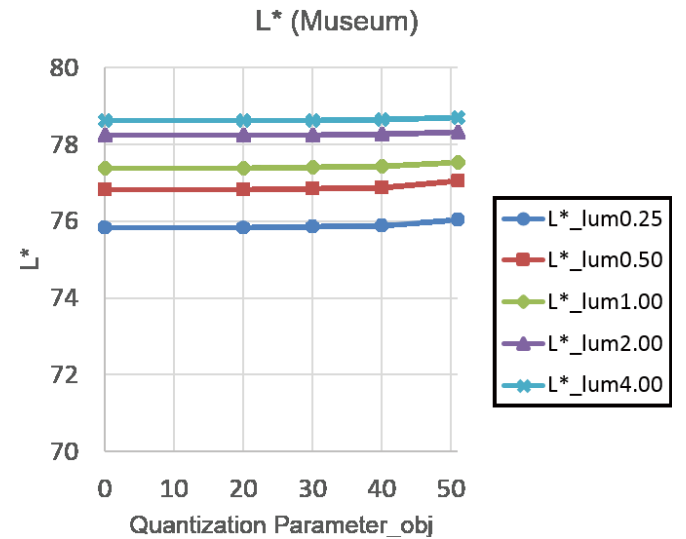

Fig. 26 Result of Exp. 3-2 (Museum)

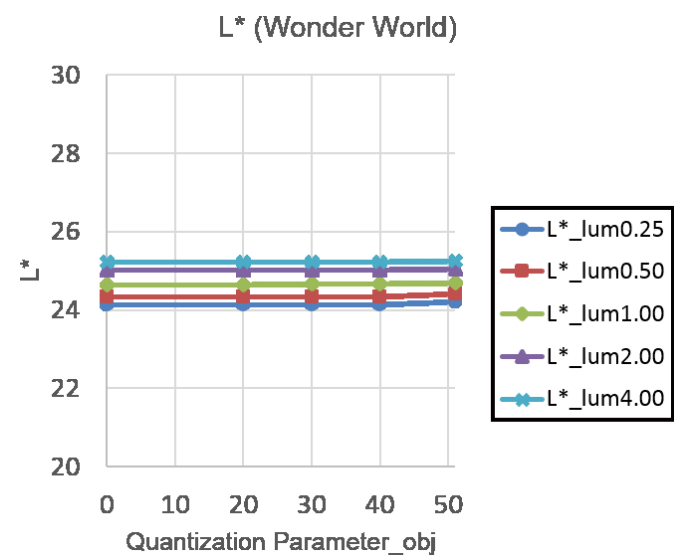

Fig. 27 Result of Exp. 3-2 (Wonder World)

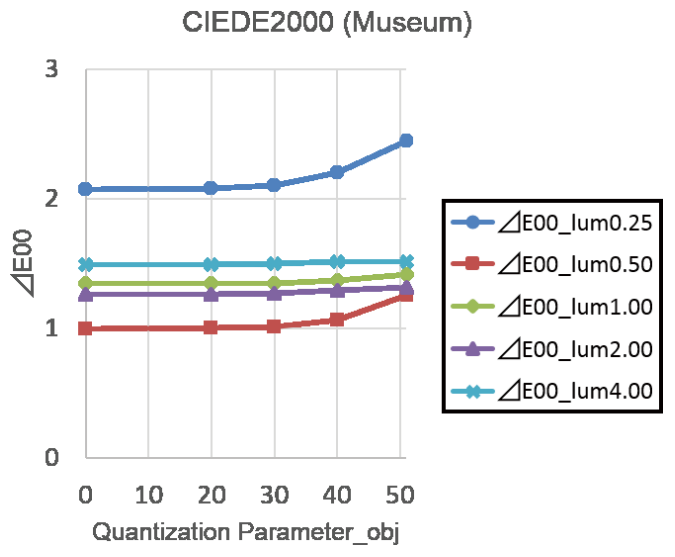

Fig. 28 Result of Exp. 3-3 (Museum)

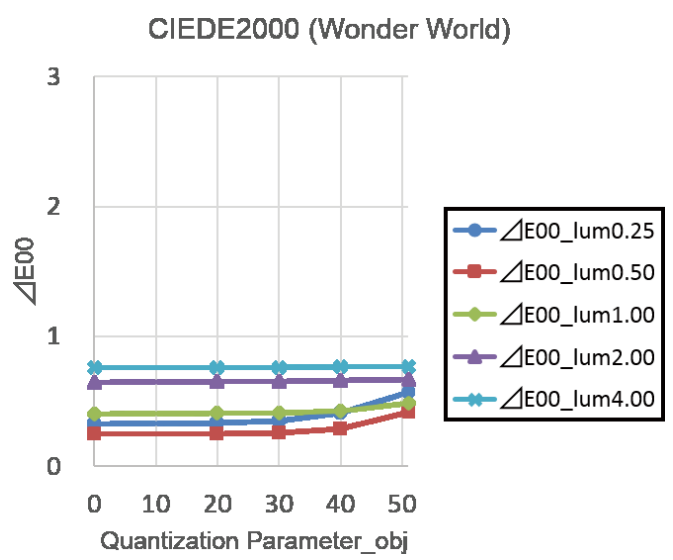

Fig. 29 Result of Exp. 3-3 (Wonder World)

the case of from Exp. 2-2 to Exp. 3-2.

\subsection{Result of Exp. 3-3}

Figures 28 and 29 show the result of Exp. 3-3 ("Museum" and "Wonder World"). The vertical axis denotes $\Delta E_{00}$ (CIEDE2000 color difference), and the horizontal axis represents $Q P$, as in the cases of Exps. 1-3 and 2-3. In Figs. 28 and 29, we observe that $\Delta E_{00 m}$ and $\Delta E_{00 w}$ represent the color difference in each image and are generated by a summation of the color difference values for the image pixels for eight viewpoints, as in the cases of Exps. 1-3 and 2-3.

From Figs. 28 and 29, we observe that $\Delta E_{00 m}$ is higher than $\Delta E_{00 w}(1-2.5 v s .0 .2-0.8)$. The difference in $\Delta E_{00 m}$ is larger than that in $\Delta E_{00 w}$. This tendency is similar to that observed in Exp. 3-2. The range of $\Delta E_{00 m}$ is larger than that of $\Delta E_{00 \mathrm{w}}$. This tendency is similar to that observed in Exp. 2-3 and implies that if we process the contrast enhancement of the object region, it is easy not for the object region of "Museum" but for the object region of "Wonder World" to affect the color difference when CIEDE2000 is used. We believe that these results are affected by the shape, color, and the area of the object region.

\subsection{Discussion}

From the result of Exp. 3, we conclude that the difference in $L^{*}$ and $\Delta E_{00}$ in the case of "Museum" was larger than those in the case of "Wonder World." Further, we believe that not only the color brightness but also the number of colors affects the evaluation value. In this study, we investigated the relationship between the luminance and the coded degradation of 3D CG images. We used the characteristic scenes for two types of 3D CG contents ("Museum," "Wonder World"). These scenes were limited for types of contents, however, we were able to obtain experimental results appropriately as a knowledge. From the SVM results, we inferred that when the contrast intensity in an image is high, the evaluation value in SVM ( Lum) tends to be high. Further, when $Q P$ has a high value, the evaluation value in SVM $(Q P)$ tends to be high. Thus, we believe that there is a relation between the luminance and the coded degradation brought about by H.265/HEVC. From the results of Exp. 31 , we can observe the steadiness of the evaluation in that the difference among the MOS patterns with a high contrast ( $L=0.25,0.50)$ is more than the difference among $M O S$ patterns that do not have a high contrast $(L=2.00,4.00)$. 
Thus, it is easy for an assessor to perceive the degradation when $L$ is low.

\section{Conclusion}

In this study, we conducted three experiments of the subjective evaluation of (1) 3D CG images, (2) the contrast enhancement focused on all the 3D CG images, (3) the contrast enhancement focused on the object region of a 3D CG image, encoded and decoded by H.265/HEVC with the eightviewpoint parallax barrier method. Then, we analyzed the obtained results by using SVM. On the other hand, we evaluated the 3D CG image objectively by using the S-CIELAB color space and CIEDE2000. From the results of three experiments, we concluded the following:

- From the result of Exp. 1, we inferred that when $Q P \geq 35$ is satisfied, $M_{M O S}$ and $W_{M O S}$ decrease dramatically and satisfy "EL." For SVM, Table 4, in class "QP," SVM correctly classification percentage, which becomes $73.8 \%$ (31/42) higher than 70\%. Therefore, we can judge "classified" in class " $Q P$." In terms of the luminance, we can see that $L_{m}^{*}$ is higher than $L_{w}^{*}$. Compared to Exp. 1-1, for the CIEDE2000 color difference, we can see that Fig. 11 has a parabolic orbit, in contrast to Fig. 9. Thus, we conclude that there is a trade-off relationship between MOS (Mean Opinion Score) and $\Delta E_{00}$ (CIEDE2000 color difference).

- From the result of Exp. 2, we can conclude that the coded degradation and the luminance between MOS and CIEDE2000 are related. We consider that it is easy not for the bright-contrast image but for the highcontrast image to perceive the coded degradation by H.265/HEVC. For SVM, from Table 7, in class "Lum," SVM correctly classification percentage, which becomes $39.2 \%$ (51/130). Therefore, we can judge "not classified." In class " $Q P$," SVM percentage, which becomes $58.4 \%$ (76/130). Therefore, we can judge "not bad classified." In terms of the luminance, we consider that it is easy not for "Wonder World" but "Museum" to change $L^{*}$. This can be seen from the range between maximum and minimum $L^{*}$. For CIEDE2000, in the case of Exp. 2-3, we cannot see as characteristic change as that in the case of Exp. 1-3. Compared to Exp. 2-2, we can see the inverse tendency. Thus, we believe that there is a trade-off relationship between $L^{*}$ (Luminance) and $\Delta E_{00}$ (CIEDE2000 color difference).

- From the result of Exp. 3, we can see the steadiness of the evaluation in that the difference among MOS patterns with a high contrast $(L=0.25,0.50)$ is more than that in patterns that do not have a high contrast ( $L=2.00,4.00)$. For SVM, percentage of class " $L u m$ " is $54.6 \%(71 / 130)$. We are able to judge "not bad classified." On the other hand, percentage of class " $Q P$ " is $57.7 \%(75 / 130)$. We are able to judge "not bad classified" same as class "Lum." For the luminance, we can obtain knowledge. For the coded degradation of the object region, we consider that it is easy not for "Wonder World" but for "Museum" to affect $L^{*}$ by the contrast enhancement processing. We can see that the $L^{*}$ range of Exp. 3-2, which is narrower than that of Exp. 2-2 without $Q P$ patterns. For CIEDE2000, if we processed the contrast enhancement of the object region, it is easy not for the object region of "Museum" but for the object region of "Wonder World" to affect by CIEDE2000. We consider that these results are affected by the shape, color, and the area of the object region.

- In general, we could verify that the SVM values of Exp. 3 tended to be higher than those of Exp. 2, and that the SVM values of Exp. 1 tended to be the highest. On the other hand, we could that the difference in $\Delta E_{00}$ (CIEDE2000 color difference) between the 3D CG image contents was clearer than that in $L^{*}$ (Luminance).

In this study, we were able to clear about the relation between coded image quality and contrast enhancement with multi-view 3D image. In the near future, if multi-view 3D TV is used widely all over the world, we expect that the results of this study, will contribute towards the establishment of an evaluation standard. If we increase the 3D contents and build a better experimental environment, this study will yield better results.

\section{Acknowledgments}

This study was carried out based on the Grant-in-Aid for Scientific Research (B) 24300046, and the research grant for Ph.D. student at Nagoya University. This paper is included improvement based on contents of our domestic conference proceeding [4], our international conference proceeding [13], and our technical report [14].

\section{References}

[1] X. Zhang, D.A. Silverstein, J.E. Farrell, and B.A. Wandell, "Color Image Quality Metric S-CIELAB and Its Application on Halftone Texture Visibility," Proc. IEEE Compcon '97, http://dx.doi.org/10.1109/CMPCON.1997.584669, February 1997.

[2] G.M. Johnson and M.D. Fairchild, "A Top Down Description of SCIELAB and CIEDE2000," Color Research \& Application, vol.28, no.6, pp.425-435, 2003.

[3] Y. Miyake and T. Nakaguchi, "Evaluation of Color Image Quality -Present State and Problems-," IEICE Fundamental Review, vol.2, no.3, pp.29-37, 2009 (in Japanese).

[4] N. Kawabata and M. Miyao, "3D CG Image Quality Evaluation Including S-CIELAB Color Space with 8 Viewpoints Glassless 3D," Proc. 2015 IEICE General Conference, A-21-2, p.292, March 2015 (in Japanese).

[5] Y. Tsuruoka and M. Meguro, "Color Image Segmentation by Using the CIEDE2000 Color-Difference formula," IEICE Tech. Rep., vol.112, no.348, SIS2012-29, pp.1-6, 2012 (in Japanese). 
[6] H. Matsumoto, K. Shibata, and Y. Horita, "Full reference image quality evaluation model considering the property of S-CIELAB color space," IEICE Tech. Rep., vol.106, no.356, CQ2006-11, pp.101-106, 2006 (in Japanese).

[7] N. Matsumoto, T. Abe, and H. Haneishi, "Evaluation Method of Degradation in Image Quality of Color Motion Pictures Due to H.264/AVC Codec (I), J J. SPIJ, vol.71, no.5, pp.368-374, May 2008 (in Japanese).

[8] N. Matsumoto, T. Abe, and H. Haneishi, "Evaluation Method of Degradation in Image Quality of Color Motion Pictures Due to H.264/AVC Codec (II),--Proposal of an Image Quality Evaluation Method That Satisfies Two Requirements," J. SPIJ, vol.72, no.4, pp.306-314, April 2009 (in Japanese).

[9] J. Bai, T. Nakaguchi, N. Tsumura, and Y. Miyake, "Evaluation of Image Corrected by Retinex Method Based on S-CIELAB and Gazing Information,” IEICE Trans. Fundamentals, vol.E89-A, no.11, pp.2955-2961, Nov. 2006.

[10] N. Kawabata and M. Miyao, "3D CG Image Quality Metrics by Regions with 8 Viewpoints Parallax Barrier Method," IEICE Trans. on Fundamentals, vol.E98-A, no.08, pp.1696-1708, Aug. 2015.

[11] Y. Kohda and T. Horiuchi, "13-11 Colorization Image Coding Using Opponent Color Space in S-CIELAB," ITE Annual Convention 2006, "13-11-1"-“13-11-2," 2006 (in Japanese).

[12] N. Kawabata and Y. Horita, "Statistical Analysis and Consideration of Subjective Evaluation of 3D CG Images with 8 Viewpoints Lenticular Lens Method," The 6th International Conference on Image Media Quality and its Applications (IMQA2013), T1-2, pp.2332, Sept. 2013.

[13] N. Kawabata and M. Miyao, "3D CG Image Quality Assessment for the Luminance Change by Contrast Enhancement Including SCIELAB Color Space with 8 Viewpoints Parallax Barrier Method," The 1st International Conference on Advanced Imaging (ICAI2015), T107-01, pp.632-635, June 2015.

[14] N. Kawabata and M. Miyao, "3D CG Image Quality Metrics for the Contrast Enhancement of the Object Region Including S-CIELAB Color Space with 8 Viewpoints Parallax Barrier Method," IEICE Tech. Rep., vol.115, no.48, IMQ2015-4, pp.17-22, May 2015.

[15] ITU-R BT.500-13: https://www.itu.int/rec/R-REC-BT.500/en, accessed Feb. 5, 2017.

[16] http://www.nict.go.jp/, accessed Feb. 5, 2017.

[17] Newsight Japan: http://newsightjapan.jp/, accessed Feb. 5, 2017.

[18] N. Kawabata and M. Miyao, "Statistical Analysis of Questionnaire Survey on the Evaluation of 3D Video Clips," IEICE Tech. Rep., vol.113, no.350, IMQ2013-22, pp.27-32, Dec. 2013 (in Japanese).

[19] C.M.Bishop, Pattern Recognition and Machine Learning, Springer Japan, 2008 (in Japanese).

[20] Weka, http://www.cs.waikato.ac.nz/ml/weka/, accessed Feb. 5, 2017.

[21] H. Abe, "Data Mining Tool: Weka," J. ITE, vol.65, no.10, pp.13981401, Oct. 2011 (in Japanese).

[22] P. Gastaldo, R. Zunino, and J. Redi, "Supporting visual quality assessment with machine learning," EURASIP Journal on Image and Video Processing, http://jivp.eurasipjournals.com/content/2013/1/54, 15 pages, 2013.

\section{Appendix A: S-CIELAB Color Space}

First, we transformed $R G B$ to $X Y Z$ space in Eq. (A. 1).

$$
\left(\begin{array}{l}
X \\
Y \\
Z
\end{array}\right)=\left(\begin{array}{lll}
0.41245 & 0.35758 & 0.18042 \\
0.21267 & 0.71516 & 0.07217 \\
0.01933 & 0.11919 & 0.95023
\end{array}\right)\left(\begin{array}{l}
R \\
G \\
B
\end{array}\right)
$$

Next, we transformed $X Y Z$ to the opponent color space, $A C_{1} C_{2}$ in Eq. (A.2).

$$
\left(\begin{array}{c}
A \\
C_{1} \\
C_{2}
\end{array}\right)=\left(\begin{array}{ccc}
0.297 & 0.72 & -0.107 \\
-0.449 & 0.29 & -0.077 \\
0.086 & -0.59 & 0.501
\end{array}\right)\left(\begin{array}{l}
X \\
Y \\
Z
\end{array}\right)
$$

Then, we carried out the convolution as a spatial filter by using Gaussian Kernel in Eqs. (A. 3), (A.4), and (A. 5). Here, Eq. (A. 3) shows the convolution. Eq. (A. 4) shows the spatial filter. Eq. (A. 5) shows the Gaussian kernel.

$$
\begin{aligned}
& \left(\begin{array}{l}
A^{\prime} \\
C_{1}^{\prime} \\
C_{2}^{\prime}
\end{array}\right)=\text { filter } *\left(\begin{array}{l}
A \\
C_{1} \\
C_{2}
\end{array}\right) \\
& \text { filter }=k \sum_{i} w_{i} E_{i} \\
& E_{i}=k_{i} \exp \left(-\frac{x^{2}+y^{2}}{\sigma_{i}^{2}}\right)
\end{aligned}
$$

After spatial filtering, we transformed the opponent color space to $X Y Z$ space in Eq. (A. 6). Here, Table A. 1 shows the Gaussian convolution kernel (weight $\left(w_{i}\right)$, spread $\left(\sigma_{i}\right)$ ).

$$
\left(\begin{array}{l}
X^{\prime} \\
Y^{\prime} \\
Z^{\prime}
\end{array}\right)=\left(\begin{array}{ccc}
0.979 & -1.535 & 0.445 \\
1.189 & 0.764 & 0.135 \\
1.232 & 1.163 & 2.079
\end{array}\right)\left(\begin{array}{l}
A^{\prime} \\
C_{1}^{\prime} \\
C_{2}^{\prime}
\end{array}\right)
$$

Then, we transformed $X Y Z$ to $L a b$ space in Eqs. (A.7), (A. 8), and (A.9).

$$
L^{*}= \begin{cases}116\left(\frac{Y}{Y_{n}}\right)^{\frac{1}{3}}-16 & \left(\frac{Y}{Y_{n}}>0.008856\right) \\ 903.3\left(\frac{Y}{Y_{n}}\right) & \text { (otherwise) }\end{cases}
$$

$$
a^{*}=\left\{\begin{array}{l}
500\left[\left(\frac{X}{X_{n}}\right)^{\frac{1}{3}}-\left(\frac{Y}{Y_{n}}\right)^{\frac{1}{3}}\right] \\
\left(\frac{X}{X_{n}}, \frac{Y}{Y_{n}}>0.008856\right) \\
500 *\left[\left[7.787\left(\frac{X}{X_{n}}\right)+\frac{16}{116}\right]-\left[7.787\left(\frac{Y}{Y_{n}}\right)+\frac{16}{116}\right]\right. \\
\text { (otherwise) }
\end{array}\right.
$$

$$
b^{*}=\left\{\begin{array}{l}
200\left[\left(\frac{Y}{Y_{n}}\right)^{\frac{1}{3}}-\left(\frac{Z}{Z_{n}}\right)^{\frac{1}{3}}\right] \\
\left(\frac{Y}{Y_{n}}, \frac{Z}{Z_{n}}>0.008856\right) \\
200 *\left[\left[7.787\left(\frac{Y}{Y_{n}}\right)+\frac{16}{116}\right]-\left[7.787\left(\frac{Z}{Z_{n}}\right)+\frac{16}{116}\right]\right] \\
\text { (otherwise) }
\end{array}\right.
$$

Table A-1 Gaussian convolution kernel [2]

\begin{tabular}{|c|c|c|}
\hline Filter & Weight $\left(w_{i}\right)$ & Spread $\left(\sigma_{i}\right)$ \\
\hline \hline Achromatic $(j=1)$ & 1.0027 & 0.0500 \\
\hline Achromatic $(j=2)$ & 0.11442 & 0.2250 \\
\hline Achromatic $(j=3)$ & -0.11769 & 7.0000 \\
\hline Red-Green $(j=1)$ & 0.61673 & 0.0685 \\
\hline Red-Green $(j=2)$ & 0.38328 & 0.8260 \\
\hline Blue-Yellow $(j=1)$ & 0.56789 & 0.0920 \\
\hline Blue-Yellow $(j=2)$ & 0.43212 & 0.6451 \\
\hline
\end{tabular}




\section{Appendix B: CIEDE2000}

CIEDE2000, which is the color difference equation, compensated the measurement result and visual appreciation as the weakness of $L^{*} a^{*} b^{*}$. First, we adjusted $a^{*}$ axis to correct for the color difference perception of low chroma colors by using Eqs. (A. 10)-(A. 12) [2].

$$
\begin{aligned}
& C_{(x, y)}^{*}=\sqrt{a_{(x, y)}^{* 2}+b_{(x, y)}^{* 2}} \\
& \bar{C}_{(x, y)}^{*}=\frac{C_{o r i g(x, y)}^{*}+C_{r e p r o(x, y)}^{*}}{2} \\
& G_{(x, y)}=0.5\left(1-\sqrt{\frac{\bar{C}_{(x, y)}^{* 7}}{\bar{C}_{(x, y)}^{* 7}+25^{7}}}\right)
\end{aligned}
$$

Equation (A.12) was used to calculate the lightness, chroma, and hue rectangular difference on a per-pixel basis for image pair as shown Eqs. (A.13)-(A. 16).

$$
\begin{aligned}
& a_{(x, y)}^{\prime}=\left(1+G_{(x, y)}\right) a_{(x, y)}^{*} \\
& L_{(x, y)}^{\prime}=L_{(x, y)}^{*} \\
& C_{(x, y)}^{\prime}=\sqrt{a_{(x, y)}^{\prime 2}+b_{(x, y)}^{\prime 2}} \\
& h_{(x, y)}^{\prime}=\arctan \left(\frac{b_{(x, y)}^{*}}{a_{(x, y)}^{\prime}}\right)
\end{aligned}
$$

In each equation, the subscript $(x, y)$ refers to the $\mathrm{x}$-y coordinates of each pixel for the image pairs. Here, we show the image pairs referred to as the original and reproduced images.

$$
\begin{aligned}
& \Delta L_{(x, y)}^{\prime}=L_{o r i g(x, y)}^{\prime}-L_{r e p r o(x, y)}^{\prime} \\
& \Delta C_{(x, y)}=C_{o r i g(x, y)}^{\prime}-C_{r e p r o(x, y)}^{\prime} \\
& \Delta h_{(x, y)}^{\prime}=h_{\text {orig }(x, y)}^{\prime}-h_{r e p r o(x, y)}^{\prime} \\
& \Delta H_{(x, y)}^{\prime}=2 \sqrt{C_{\text {orig }(x, y)}^{\prime} C_{r e p r o(x, y)}^{\prime}} \cdot \sin \left(\frac{\Delta h_{(x, y)}^{\prime}}{2}\right)
\end{aligned}
$$

If the hue angles reside in different hue quadrants, we need to be careful in order to calculating hue angle differences between the original and reproduced images. If the absolute difference between the two hue angles is greater than 180 degrees, it is important to add 360 degrees to the smaller of hue angles as shown in Eq. (A.21).

$$
\begin{cases}\text { if } & \left|\Delta h_{(x, y)}^{\prime}\right|>180, \\ \text { then } & \min \left(h_{\text {orig }(x, y)}^{\prime}, h_{\text {repro }(x, y)}^{\prime}+360\right)\end{cases}
$$

$$
\begin{aligned}
& \begin{cases}\text { if } & \Delta h_{(x, y)}^{\prime}>180 \\
\text { then } & \Delta h_{(x, y)}^{\prime}=\Delta h_{(x, y)}^{\prime}-360 \\
\text { elseif } & \Delta h_{(x, y)}^{\prime}<-180 \\
\text { then } & \Delta h_{(x, y)}^{\prime}=\Delta h_{(x, y)}^{\prime}+360\end{cases} \\
& \bar{L}_{(x, y)}^{\prime}=\frac{L_{\text {orig }(x, y)}^{\prime}+L_{r e p r o(x, y)}^{\prime}}{2}
\end{aligned}
$$

$$
\bar{C}_{(x, y)}^{\prime}=\frac{C_{\text {orig(x,y) }}^{\prime}+C_{r e p r o(x, y)}^{\prime}}{2}
$$

$$
\bar{h}_{(x, y)}^{\prime}=\frac{h_{\text {orig }(x, y)}^{\prime}+h_{\text {repro }(x, y)}^{\prime}}{2}
$$

The lightness and chroma functions, $S_{L}, S_{C}$ are shown in Eqs. (A. 26) and (A.27).

$$
\begin{aligned}
& S_{L(x, y)}^{\prime}=1+\frac{0.015\left(\bar{L}_{(x, y)}^{*}-50\right)^{2}}{\sqrt{20+\left(\bar{L}_{(x, y)}^{*}-50\right)^{2}}} \\
& S_{C(x, y)}^{\prime}=1+0.045 \bar{C}_{(x, y)}^{\prime}
\end{aligned}
$$

The hue angle dependency can be calculated using Eq. (A.28). And then, the chroma dependency can be calculated using Eq. (A-29).

$$
\begin{aligned}
T_{(x, y)}= & 1-0.17 \cos \left(\bar{h}_{(x, y)}^{\prime}-30\right)+0.24 \cos \left(2 \bar{h}_{(x, y)}^{\prime}\right) \\
& +0.32 \cos \left(3 \bar{h}_{(x, y)}^{\prime}+6\right)-0.20 \cos \left(4 \bar{h}_{(x, y)}^{\prime}-63\right)
\end{aligned}
$$

$$
S_{H(x, y)}^{\prime}=1+0.015 \bar{C}_{(x, y)}^{\prime} \cdot T_{(x, y)}
$$

Equation (A. 30) shows the rotation function used for compensating for the blue region of CIELAB.

$$
R_{C(x, y)}=2.0 \sqrt{\frac{\bar{C}_{(x, y)}^{7}}{\bar{C}_{(x, y)}^{7}+25^{7}}}
$$

Equation (A. 31) shows a hue angle dependency. 
$\Delta \theta_{(x, y)}=30 \exp \left(-\left(\bar{h}_{(x, y)}^{\prime}-\frac{275^{\circ}}{25}\right)^{2}\right)$

The rotation function shows Eq. (A. 32).

$$
R_{T(x, y)}=-\sin \left(2 \Delta \theta_{(x, y)}\right) R_{C(x, y)}
$$

For $L^{*} a^{*} b^{*}$ of both original image and coding image in obtained in Eqs. (A.7), (A. 8), and (A.9) of Appendix A, when we calculated the additional weighting coefficient $\left(S_{L}, S_{C}, S_{H}\right)$ and parametric coefficient $\left(K_{L}, K_{C}, K_{H}\right)$ based on the difference of the luminance, intensity, and hue, the color difference $\Delta E_{00}$ for each pixel could be represented as Eq. (A. 33) from Eq. (A. 34) [2].

$$
\begin{aligned}
& \Delta E_{00}=\sqrt{L^{2}+C^{2}+H^{2}+\left(R_{T} C H\right)} \\
& L=\frac{\Delta L^{\prime}}{K_{L} \cdot S_{L}}, C=\frac{\Delta C^{\prime}}{K_{C} \cdot S_{C}}, H=\frac{\Delta H^{\prime}}{K_{H} \cdot S_{H}}
\end{aligned}
$$

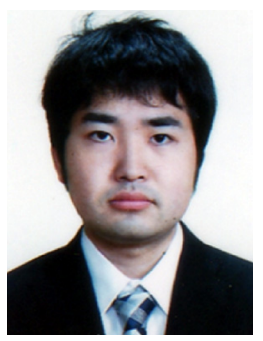

Norifumi Kawabata received the B.E., M.E. degrees in Intellectual Information Engineering from University of Toyama, Japan, in 2011, and 2013, respectively. Since 2013, he is a $\mathrm{Ph}$.D. student in Department of Information Engineering, Graduate School of Information Science in Nagoya University. He got a prize of "IEICE Tokai Section Student Award 2014" in June 2015. He is mainly interested in multi-view 3D image quality, digital watermarking, image processing, and human interface interaction.

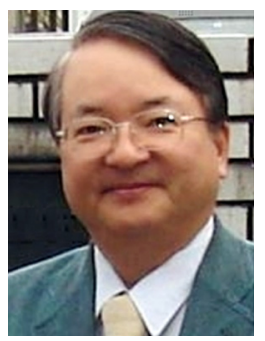

Masaru Miyao received the B.M., Ph.D. degrees in social medicine, department of medicine from Nagoya University, Japan, in 1977 and 1985, respectively. He is currently a professor in Department of Information Engineering, Graduate School of Information Science in Nagoya University. He is mainly interested in public health, social medicine, industrial medicine, health care information, medical statistics, human interface, usability, 3D image, and ergonomics. 